\title{
LINEAR TRANSFORMATIONS BETWEEN HILBERT SPACES AND THE APPLICATION OF THIS THEORY TO LINEAR PARTIAL DIFFERENTIAL EQUATIONS*
}

\author{
BY
}

F. J. MURRAY

\section{INTRODUCTION}

Let us consider the equation

(A)

$$
\begin{aligned}
L(u) \equiv & A_{2,0} \frac{\partial^{2} u}{\partial x^{2}}+A_{1,1}\left(\frac{\partial^{2} u}{\partial x \partial y}+\frac{\partial^{2} u}{\partial y \partial x}\right)+A_{0,2} \frac{\partial^{2} u}{\partial y^{2}}+A_{1,0} \frac{\partial u}{\partial x} \\
& +A_{0,1} \frac{\partial u}{\partial y}+A_{0,0} u=v,
\end{aligned}
$$

where the $A$ 's and $u$ are complex-valued functions, such that the above expression has a meaning on a bounded connected region $S$ in the real $X Y$ plane. We assume that the $A$ 's are bounded measurable functions on $S$. We shall restrict $u$ in such a manner that (A) may be considered a linear transformation between two Hilbert spaces.

In the first five sections of this paper, we study the linear transformations between two abstract Hilbert spaces $\mathfrak{W}_{1}$ and $\mathfrak{W}_{2}$, which are regarded as coinciding only in special cases. While our investigations are naturally based on the modern work $\dagger$ on the subject, they are particularly closely allied to a paper of J. von Neumann. $\ddagger$ Roman numerals indicate essentially new results.

In $\$ 1$ and $\S 2$, we adapt the treatment of $(\mathrm{N})$ to our problem to obtain the elementary theory of such transformations. In $\$ 2$, we also discuss the significance in terms of groups of the adjoint. $\$ 3$ deals with continuous linear transformations and while most of these results are well known,\| Theorem $I$ is new and is used later. It also can be considered as indicating the "graphical interpretation" of certain general results of J. von Neumann, which are cited in connection with the theorem. Theorem II deals with the solution of

* Presented to the Society, March 31, 1934; received by the editors April 17 and May 3, 1934.

$\dagger$ Cf. Stone, Linear Transformations in Hilbert Space, American Mathematical Societ.y Colloquium Publications, vol. XV, New York, 1932, also Banach, Théorie des Opérations Linérıires, Warsaw, 1932. These monographs will be denoted by $(S)$ and (B) respectively.

$\ddagger$ Annals of Mathematics, (2), vol. 33 (1932), pp. 294-310. We will refer to this Memoir by (N).

II Cf. (B), Chap. I, p. 23, Chap. III, p. 37, and Chap. VI, p. 100. 
the equation $T f=g$ and the note on constructions indicates the computational methods to be used.

We define "breakage" in $§ 5$. The theory of the manifolds which break a closed linear transformation, with domain everywhere dense in $\mathfrak{S}_{1}$, is shown to be completely analogous to the theory of the manifolds which reduce a selfadjoint transformation. Considerations of breakage lead to methods which, in a certain sense, compensate in the study of inverses of contractions of a closed linear transformation for the lack of compactness of Hilbert space.

In the remaining sections, we return to the consideration of (A). In $\$ 6$, we set up a Hilbert space $\mathfrak{B}$, in such a manner that (A) can be considered as a limited transformation from $\mathfrak{B}$ to $\mathfrak{\Omega}_{2}(\$ 7)$. In $\S 8$, we apply Theorems 1.16 and II, to obtain a generalization of the well known methods of Ritz. ${ }^{*}$ In the remaining sections we show that it is possible to use the same notions, concerning transformations between Hilbert spaces, to solve a quite general type of boundary problem (cf. $\$ 10)$. Certain restrictions on the boundary of $S$ are used and these are given in $\$$.

Our methods apply to partial differential equations as such and we are not restricted to differential systems as previous writers have been, who use Hilbert space. $\dagger$ In this connection, it may be pointed out that while in the methods used here it is not necessary to take into account the distinction between elliptic, hyperbolic, and parabolic equations, nevertheless when these methods are applied, the results are essentially different. For instance, examples indicate that if the expression $L(u)$ is elliptic, the range of the transformation $T$ of $\$ 7$ is the whole space $\mathfrak{R}_{2}$ and that this is not true in general if $L(u)$ is non-elliptic, although the range of $T$ may be everywhere dense in $\mathfrak{R}_{\mathbf{2}}$. See examples in $\$ 8$.

\section{General Definitions AND theorems}

The present section is devoted to general definitions and theorems. A knowledge of the definitions and elementary theorems of Hilbert space is assumed. (Cf. e.g. (S), Chap. 1.) We shall employ Stone's notation systematically. We shall let $(, \quad)_{1},(, \quad)_{2}$ stand for the inner products in $\mathfrak{S}_{1}$ and $\mathfrak{S}_{2}$ respectively.

Definition 1.1. By the (cartesian) product, $\mathfrak{S}_{1} \times \mathfrak{S}_{2}$, of the Hilbert spaces $\mathfrak{S}_{1}$ and $\mathfrak{S}_{2}$ (distinct or not), is meant the space of all ordered pairs $\left\{f_{1}, f_{2}\right\}$, $f_{1} \in \mathfrak{S}_{1}, f_{2} \in \mathfrak{S}_{2}$, with the linear operations $(+, a \cdot)$ and the inner product $(,)_{1,2}$ defined by the equations

* Journal für Mathematik, vol. 135 (1908-09), pp. 1-61. index).

$\dagger$ For an application of the methods of this space to differential equations, compare (S) (see 


$$
\begin{aligned}
\left\{f_{1}, f_{2}\right\}+\left\{g_{1}, g_{2}\right\} & =\left\{f_{1}+g_{1}, f_{2}+g_{2}\right\} ; \\
a \cdot\left\{f_{1}, f_{2}\right\} & =\left\{a f_{1}, a f_{2}\right\} ; \\
\left(\left\{f_{1}, f_{2}\right\},\left\{g_{1}, g_{2}\right\}\right)_{1,2} & =\left(f_{1}, g_{1}\right)_{1}+\left(f_{2}, g_{2}\right)_{2} .
\end{aligned}
$$

It is known that the space $\mathfrak{S}_{1} \times \mathfrak{S}_{2}$ is a Hilbert space (cf. (S), Theorem 1.26). To every pair $\left\{f_{1}, f_{2}\right\} \epsilon \mathfrak{S}_{1} \times \mathfrak{S}_{2}$ we make correspond the pair $\left\{f_{2}, f_{1}\right\} \in \mathfrak{S}_{2} \times \mathfrak{W}_{1}$ and if $S \subseteq \mathfrak{S}_{1} \times \mathfrak{S}_{2}$ is given we denote by $S^{-1}$ the set which is in $\mathfrak{W}_{2} \times \mathfrak{W}_{1}$ and whose elements correspond to elements in $S$.

We now introduce the notion of a many-one correspondence between a set $\mathfrak{D} \subseteq \mathfrak{S}_{1}$ and a set $\mathfrak{R} \subseteq \mathfrak{S}_{2}$ in abstract form.

DEFINITION 1.2. Let $\mathfrak{T}$ be a non-empty subset of $\mathfrak{S}_{1} \times \mathfrak{S}_{2}$, no two of the ordered pairs of $\mathfrak{T}$ having the same first element. Then $\mathfrak{T}$ is called a transformation from $\mathfrak{W}_{1}$ to $\mathfrak{S}_{2}$; when regarded as carrying the first element $f_{1}$ of each pair $\left\{f_{1}, f_{2}\right\} \in \mathfrak{T}$ into the second $f_{2}$, it is denoted by $T: f_{2}=T f_{1}$ (T being obviously "single-valued"). By the domain $\mathfrak{D}$ of $\mathfrak{T}$ (or $T)$ is meant the class of first elements, and by the range $\Re$ of $\mathfrak{T}$, the class of second elements of the class of ordered pairs $\mathfrak{T}$. We also write $\Re=T \mathfrak{D}$.

When several transformations $\left(T_{1}, T_{2}, T^{\prime}, T^{*}\right.$, etc.) are studied, their respective domains, ranges, etc., are designated with corresponding marks $\left(\mathfrak{D}_{1}, \mathfrak{D}_{2}, \mathfrak{D}^{\prime}, \mathfrak{D}^{*}\right.$, etc., $\Re_{1}, \Re_{2}, \mathfrak{R}^{\prime}, \mathfrak{R}^{*}$, etc., $\mathfrak{T}_{1}, \mathfrak{I}_{2}, \mathfrak{T}^{\prime}, \mathfrak{T}^{*}$, etc.).

Definition 1.3. Let $T_{1}$ and $T_{2}$ be two transformations from $\mathfrak{W}_{1}$ to $\mathfrak{W}_{2} ;$ (a) if $\mathfrak{I}_{1}=\mathfrak{I}_{2}$, we write $T_{1}=T_{2}$, then $\mathfrak{D}_{1}=\mathfrak{D}_{2}$ and $\Re_{1}=\Re_{2} ;(\mathrm{b})$ if $\mathfrak{D}_{1} \cdot \mathfrak{D}_{2} \neq 0, T_{1}+T_{2}$ shall be the transformation (cf. Definition 1.2) corresponding to the set $\mathfrak{T} \subseteq \mathfrak{S}_{1} \times \mathfrak{S}_{2}$, defined as follows: $\mathfrak{I}$ is the class of all pairs $\left\{f_{1}, f_{2}\right\}$ such that $f_{1} \in \mathfrak{D}_{1} \cdot \mathfrak{D}_{2}$ and $f_{2}=\mathfrak{T}_{1} f_{1}+T_{2} f_{1}$; (c) if a is a scalar, aT $T_{1}$ shall correspond to the class $\mathfrak{T}$ of all pairs $\left\{f_{1}, a f_{2}\right\} \in \mathfrak{S}_{1} \times \mathfrak{W}_{2}$, such that $\left\{f_{1}, f_{2}\right\} \in \mathfrak{T}_{1}$. By Definition 1.2, $a T_{1}$ is a transformation.

Definition 1.4. Let $\mathfrak{S}_{1}, \mathfrak{S}_{2}, \mathfrak{S}_{3}$ be three Hilbert spaces (distinct or not) and let $T_{1}$ and $T_{2}$ denote transformations from $\mathfrak{S}_{1}$ to $\mathfrak{S}_{2}$ and from $\mathfrak{W}_{2}$ to $\mathfrak{S}_{3}$, respectively, corresponding with the respective sets $\mathfrak{I}_{1} \subseteq \mathfrak{S}_{1} \times \mathfrak{W}_{2}$ and $\mathfrak{I}_{2} \subseteq \mathfrak{V}_{2} \times \mathfrak{W}_{3}$. Suppose furthermore $\Re_{1} \cdot \mathfrak{D}_{2} \neq 0$. Then the product $T=T_{2} T_{1}$ shall denote the transformation from $\mathfrak{S}_{1}$ to $\mathfrak{S}_{3}$, determined by the set $\mathfrak{T} \subseteq \mathfrak{S}_{1} \times \mathfrak{S}_{3}$, defined in the following manner. $\mathfrak{T}$ shall be the class of all pairs $\left\{f_{1}, f_{3}\right\} \in \mathfrak{S}_{1} \times \mathfrak{W}_{3}$ for which there exists an element $f_{2} \in \Re_{1} \cdot \mathfrak{D}_{2}\left(\subseteq \mathfrak{S}_{2}\right)$ such that $\left\{f_{1}, f_{2}\right\} \in \mathfrak{T}_{1}$ and $\left\{f_{2}, f_{3}\right\} \in \mathfrak{T}_{2}$.

It should be observed that the symbol $T_{1} T_{2}$ has no meaning in general, and even in the special case where $\mathfrak{S}_{1}=\mathfrak{S}_{2}=\mathfrak{S}_{3}$ and $T_{1} T_{2}$ can exist, it is, in general, distinct from $T_{2} T_{1}$. 
Definition 1.5. The notation $T_{1} \supseteq T_{2}$ and $T_{1} \supset T_{2}$ is used to signify that $\mathfrak{I}_{1} \supseteq \mathfrak{I}_{2}$ and $\mathfrak{I}_{1} \supset \mathfrak{I}_{2}$, respectively. In the former case $T_{1}$ is said to be an extension of $T_{2}, T_{2}$ a contraction of $T_{1}$, or the contraction of $T_{1}$ with domain $\mathfrak{D}_{2}$; in the latter case $T_{1}$ is said to be a proper extension of $T_{2}, T_{2}$ a proper contraction of $T_{1}$.

DeFINITION 1.6. Let the transformation $T$ from $\mathfrak{W}_{1}$ to $\mathfrak{W}_{2}$ be such that the set $\mathfrak{I}$ contains no two pairs with the same last elements. Then $T$ is said to have an inverse $T^{-1}$, which is defined by the set $\mathfrak{T}^{-1} \subseteq \mathfrak{W}_{2} \times \mathfrak{W}_{1}$, consisting of the pairs in $\mathfrak{T}$, with their order inverted.

Note the obvious relations

$$
\mathfrak{D}^{-1}=\Re ; \Re^{-1}=\mathfrak{D} ; T T^{-1} \subseteq I, T^{-1} T \subseteq I .
$$

Definition 1.7. A sequence of transformations $\left\{T_{n}\right\}$ from $\mathfrak{W}_{1}$ to $\mathfrak{S}_{2}$, with domains $\left\{\mathfrak{D}_{n}\right\}$, is said to converge on the set $\mathbb{S}$ if

(1) $\subseteq \subseteq \lim \inf \mathfrak{D}_{n} ; *$

(2) for every $f_{\epsilon} \subseteq$, the sequence $\left\{f, T_{n} f\right\}$ converges.

$A$ sequence of transformations $\left\{T_{n}\right\}$, from $\mathfrak{W}_{1}$ to $\mathfrak{W}_{2}$, is said to have the limit $T$ on $\mathfrak{S}$ if

(1) $\subseteq \subseteq\left(\lim \inf \mathfrak{D}_{n}\right) \cdot \mathfrak{D}$;

(2) for every $f_{\epsilon} \subseteq$, the sequence $\left\{f, T_{n} f\right\}$ converges to $\{f, T f\}$.

We shall write $T_{n} \rightarrow T, n \rightarrow \infty$ on $\subseteq$.

Since Hilbert space is complete, it is easy to show

THEOREM 1.1. If a sequence $\left\{T_{n}\right\}$ from $\mathfrak{W}_{1}$ to $\mathfrak{W}_{2}$ converges on $\mathfrak{S}$, then there exists a transformation $T$ from $\mathfrak{W}_{1}$ to $\mathfrak{W}_{2}$, such that $T_{n} \rightarrow T, n \rightarrow \infty$ on $\mathfrak{S}$. If $T_{n} \rightarrow T$ on $\mathfrak{S}$, then the sequence $\left\{T_{n}\right\}$ is convergent on $\mathfrak{S}$.

Definition 1.8. A transformation $T$ from $\mathfrak{S}_{1}$ to $\mathfrak{W}_{2}$ is said to be continuous at an element $f$ in its domain, if to each positive number $\epsilon$, there corresponds a positive number $\delta=\delta(\epsilon, f)$ such that whenever $g$ is an element of $\mathfrak{D}$, satisfying the inequality $\|f-g\|_{1} \leqq \delta$, the element $T g$ satisfies the inequality $\|T f-T g\|_{2} \leqq \epsilon$. $A$ transformation $T$ is said to be continuous, if it is continuous at every element in its domain.

Definition 1.9. A transformation $T$ from $\mathfrak{W}_{1}$ to $\mathfrak{W}_{2}$ is said to be closed if $\mathfrak{T}$ is closed.

Definition 1.10. A transformation $T$ from $\mathfrak{W}_{1}$ to $\mathfrak{W}_{2}$ is said to be linear if $\mathfrak{T}$ is a linear manifold.

* $\lim \inf \mathfrak{D}_{n}$ is the set of points, each of which is in all but a finite number of the ("almost every") $\mathfrak{D}_{n}$. 
We state the following theorems the proofs of which are easy and may be omitted.

THEOREM 1.2. The domain and range of a linear transformation are linear manifolds.

THEOREM 1.3. If $T_{1}$ and $T_{2}$ are two linear transformations from $\mathfrak{S}_{1}$ to $\mathfrak{W}_{2}$, then $a T_{1}$ and $T_{1}+T_{2}$ are linear transformations.

THEOREM 1.4. If $T_{2}$ is a linear transformation from $\mathfrak{W}_{1}$ to $\mathfrak{S}_{2}$ and $T_{1}$ is a linear transformation from $\mathfrak{S}_{2}$ to $\mathfrak{S}_{3}$, then $T=T_{1} T_{2}$ is linear.

THEOREM 1.5. If $T$ is a linear transformation possessing an inverse then $T^{-1}$ is linear. If $T$ is a closed transformation possessing an inverse, then $T^{-1}$ is closed.

THEOREM 1.6. If $T$ is a transformation, any subset of $\mathfrak{T}$ is a transformation.

THEOREM 1.7. If the transformation $T$ has a linear extension then there exists a unique linear transformation $\hat{T}$ such that

(1) $\hat{T}$ is an extension of $T$;

(2) if $T^{\prime}$ is linear and $T^{\prime} \supseteq T$, then $T^{\prime} \supseteq \hat{T}$.

If $T$ has a closed linear extension then there exists a unique closed linear transformation $\tilde{T}$ such that

(1) $\tilde{T}$ is an extension of $T$ and $\hat{T}$;

(2) if $T^{\prime}$ is a closed linear extension of $T, T^{\prime} \subseteq \tilde{T}$.

If $T$ has a (closed) linear extension $T_{0}$, we take for $\hat{T}$ (or $\tilde{T}$ ) the (closed) linear manifold determined by $\mathfrak{T}$. The theorem follows immediately from Theorem 1.6, Definitions 1.8 and 1.9 above, and (S) Definition 1.4.

\section{ThE PERPENDICULAR AND ADJOINT OF A TRANSFORMATION}

We return to the study of perfectly general transformations $T_{1}$ from $\mathfrak{W}_{1}$ to $\mathfrak{W}_{2}$ and $T_{2}$ from $\mathfrak{W}_{2}$ to $\mathfrak{S}_{1}$, determined by the sets $\mathfrak{T}_{1}$ and $\mathfrak{I}_{2}$ in $\mathfrak{S}_{1} \times \mathfrak{Q}_{2}$ and $\mathfrak{W}_{2} \times \mathfrak{S}_{1}$ respectively While our treatment paraphrases the work of $(\mathrm{N})$, we wish to call attention to the simplicity in conceptions and proofs obtained by studying directly not the adjoint but the perpendicular to a transformation, which notion we define as follows.

DEFINITION 1.11. The transformations $T_{1}$ from $\mathfrak{W}_{1}$ to $\mathfrak{W}_{2}$, and $T_{2}$ from $\mathfrak{\bigvee}_{2}$ to $\mathfrak{S}_{1}$, shall be said to be perpendicular (symbolically $T_{1} \perp T_{2}$ ) if $\mathfrak{T}_{1}^{-1}$ and $\mathfrak{T}_{2}$ are orthogonal in $\mathfrak{S}_{2} \times \mathfrak{S}_{1}$. They shall be said to be adjoint $\left(T_{1} \wedge T_{2}\right)$, provided that $T_{1}$ and $-T_{2}$ are perpendicular.

Obviously $T_{1} \perp T_{2}$ implies $T_{2} \perp T_{1}$; also $T_{1} \perp T_{2}$ implies that, for all pairs $\left\{f, T_{1} f\right\} \mathfrak{T}$ and $\left\{T_{2} g, g\right\} \in \mathfrak{T}_{2}$, 


$$
0=\left(\left\{f, T_{1} f\right\},\left\{T_{2} g, g\right\}\right)_{1,2}=\left(f, T_{2} g\right)_{1}+\left(T_{1} f, g\right)_{2},
$$

and conversely this equation, valid for all $f \in \mathfrak{D}_{1}$ and $g \in \mathfrak{D}_{2}$, implies that $T_{1} \perp T_{2}$. We are immediately led to the following theorem which connects our definition with the usual one. $\dagger$

THEOREM 1.8. The relation $T_{1} \wedge T_{2}$ will hold if and only if

$$
\left(f, T_{2} g\right)_{1}=\left(T_{1} f, g\right)_{2}
$$

for all $f \in \mathfrak{D}_{1}$, and $g \in \mathfrak{D}_{2}$, and further $T_{1} \wedge T_{2}$ implies $T_{2} \wedge T_{1}$.

We now suppose given a perfectly general transformation $T$ from $\mathfrak{Q}_{1}$ to $\mathfrak{W}_{2}, \mathfrak{T}$ and $\mathfrak{D}$ being as usual, and consider the problem of finding transformations perpendicular to it. We are led to the following theorem.

THEOREM 1.9. Let $\mathfrak{T}^{\perp}$ be the class of all elements of $\mathfrak{W}_{2} \times \mathfrak{S}_{1}$, orthogonal to $\mathfrak{T}^{-1}$. Then a necessary and sufficient condition that $\mathfrak{T}^{\perp}$ constitute a transformation from $\mathfrak{W}_{2}$ to $\mathfrak{S}_{1}$, is that $\mathfrak{D}$ span $\mathfrak{W}_{1} . \ddagger$ When $\mathfrak{D}$ spans $\mathfrak{S}_{1}$, then $T \perp T^{\prime}$ if and only if $\mathfrak{T}^{\prime} \subseteq \mathfrak{T}^{\perp}$.

The proof is quite analogous to that of (S) Theorem 2.6.

DEFINITION 1.12. When the transformation $T$ from $\mathfrak{S}_{1}$ to $\mathfrak{W}_{2}$ is such that $\mathfrak{D}$ spans $\mathfrak{\bigvee}_{1}$, then $T^{\perp}$ defined by the set $\mathfrak{T}^{\perp}$, of Theorem 1.9 , is called the perpendicular of $T$, and $T^{*}=-T \perp$ is called the adjoint of $T . \S$

The following Theorems are capable of very simple proofs which we omit.

THEOREM 1.10. If $T^{\perp}$ exists, then $\mathfrak{T}^{\perp}$ is the orthogonal complement in $\mathfrak{S}_{2} \times \mathfrak{W}_{1}$ of the closed linear manifold determined by $\mathfrak{I}^{-1}$.

THEOREM 1.11. If $T^{\perp}$ exists, then it is a closed linear transformation.

THEOREM 1.12. If $T$ is a transformation from $\mathfrak{W}_{1}$ to $\mathfrak{W}_{2}$, whose domain determines $\mathfrak{W}_{1}$, and whose range determines $\mathfrak{W}_{2}$, and if $T$ possesses an inverse, then $T^{\perp}$ and $\left(T^{-1}\right) \perp$ are inverses.

$\mathfrak{I}^{\perp}$ and $\left(\mathfrak{T}^{-1}\right)^{\perp}$ correspond to transformations by Theorem 1.9 and it is easy to verify that $\left(\mathfrak{I}^{\perp}\right)^{-1}=\left(\mathfrak{I}^{-1}\right)^{\perp}$, since the correspondence $\mathfrak{A} \sim \mathfrak{A}^{-1}$ preserves orthogonality.

† Cf. (S) Definition 2.7, (B) p. 99.

$\ddagger$ The closed linear manifold determined by $\mathfrak{D}$ is $\mathfrak{S}_{1}$.

$\S$ For limited transformations (cf. \$4), in view of Theorem 1.8, this definition is equivalent to that given by (B) p. 99. Remembering that a Hilbert space is self-dual, we see that to every linear functional on $\mathfrak{W}_{2}, Y$ or on $\mathfrak{W}_{1}, X$, we can make correspond respectively an $f \in \mathfrak{F}_{2}$ and an $f^{*} \mathfrak{S}_{1}$ such that $Y(h)=(h, f)_{2} ; X(g)=\left(g, f^{*}\right)_{2}$ for all $h$ in $\mathfrak{S}_{2}$ and all $g$ in $\mathfrak{S}_{1}$. Thus when $T$ is limited and $X=\bar{T} Y$ then $\left(g, f^{*}\right)_{1}=X(g)=\bar{T}(Y)(g)=Y(T g)=(T g, f)_{2}$ or $f^{*}=T^{*} f$. 
THEOREM 1.13. A necessary and sufficient condition that a transformation $T$ from $\mathfrak{W}_{1}$ to $\mathfrak{W}_{2}$, with domain everywhere dense in $\mathfrak{W}_{1}$, should have a closed linear extension is that the domain of $T^{\perp}$ (and hence of $T^{*}$ ) be everywhere dense in $\mathfrak{\$}_{2}$. If $\tilde{T}$ exists then $\tilde{T}=\left(T^{\perp}\right) \perp=-\left(-T^{*}\right)^{*}=\left(T^{*}\right)^{*}$.

The proofs of this theorem and the three following are quite similar to the proofs of the corresponding theorenus in the memoir of von Neumann.

THEOREM 1.14. Let $T$ be a closed linear transformation from $\mathfrak{W}_{1}$ to $\mathfrak{W}_{2}$, with domain everywhere dense in $\mathfrak{S}_{1}$. Then $T^{\perp}$ and $T^{*}$ are closed and linear and their common domain is everywhere dense in $\mathfrak{S}_{2}$, and $T^{*} T$ is self-adjoint. $\dagger$

THEOREM 1.15. Let $T$ be as in Theorem 1.14. Let $\mathfrak{D}_{1}$ be the domain of $T^{*} T$ and $T_{1}$ the contraction of $T$ with domain $\mathfrak{D}_{1}$. Then $\tilde{T}_{1}=T$.

THEOREM 1.16. Let $T$ be as in Theorem 1.14. Let the linear manifold of all $f \in \mathfrak{W}_{1}$ such that $T f=0$ be denoted by $\mathfrak{R}$ and the linear manifold of all $f \in \mathfrak{W}_{2}$ such that $T^{*} f=0$ be denoted by $\mathfrak{N}^{*}$; then $\mathfrak{N}$ and $\mathfrak{N}^{*}$ are closed, and if $\overline{\mathfrak{M}}(\mathfrak{R})$ and $\overline{\mathfrak{M}}\left(\Re^{*}\right)$ denote the closed linear manifolds determined by $\Re$ and $\mathfrak{R}^{*}$, respectively, then

$$
\overline{\mathfrak{M}}\left(\mathfrak{R}^{*}\right)=\mathfrak{W}_{1} \ominus \mathfrak{R} ; \quad \overline{\mathfrak{M}}(\mathfrak{R})=\mathfrak{E}_{2} \ominus \mathfrak{N}^{*} .
$$

A Hilbert space is of course an Abelian group with operators (i.e., multiplication by scalars) $\ddagger$ A linear manifold $\mathfrak{M}$ is an Abelian subgroup of $\mathfrak{E}$ and hence we can form the quotient group $\mathfrak{E} / \mathfrak{M}$, i.e., the group of the remainder classes of $\mathfrak{E}, \bmod \mathfrak{M} . \S$ Now if $\mathfrak{M}$ is closed, $\mathfrak{S} \Theta \mathfrak{M}$ is simply isomorphic to $\mathfrak{E} / \mathfrak{M}$ under the correspondence which links $f \in \mathfrak{W} \ominus \mathfrak{M}$ with the remainder class, which consists of all elements congruent to $f$, $\bmod \mathfrak{M}$.

Let $\mathfrak{I}$ correspond to a closed linear transformation from $\mathfrak{W}_{1}$ to $\mathfrak{W}_{2}$. $\mathfrak{I}^{\perp} \subseteq \mathfrak{V}_{2} \times \mathfrak{W}_{1}$ is simply isomorphic to the quotient group of $\mathfrak{I}^{-1}$. These notions persist even when considered in linear vector space \& more general than Hilbert space where, however, $\mathfrak{I}^{\perp}$ is isomorphic with the set of linear functionals on $\mathbb{E}_{1} \times \mathbb{E}_{2} / \mathfrak{T}^{-1}$.

\section{CONTINUOUS LINEAR TRANSFORMATIONS}

We now consider continuous linear transformations. Where proofs are omitted the theorems are either special cases of theorems in (B) or their proofs are quite analogous to the proofs of corresponding theorems in (S).

THEOREM 1.17. If a linear transformation $T$ from $\mathfrak{S}_{1}$ to $\mathfrak{S}_{2}$ is continuous at one element in its domain it is uniformly continuous at each point in its domain.

$\dagger$ Cf. (S) 2.11. A transformation (in $\mathfrak{S}$ ) is said to be self-adjoint if it is equal to its adjoint.

$\ddagger$ Cf. van der Waerden, Höhere Algebra, vol. 1, chap. 6, Springer, 1932.

$\S$ Cf. van der Waerden, loc. cit., vol. 1, pp. 31-36, 132-135. 
THEOREM 1.18. If $T$ is a linear transformation from $\mathfrak{W}_{1}$ to $\mathfrak{W}_{2}$, then $T$ is continuous if and only if there is a positive number $C$ such that for all $f \in \mathfrak{D}$,

$$
\|T f\|_{2} \leqq C\|f\|_{1} .
$$

Definition 1.13. A linear transformation $T$ from $\mathfrak{W}_{1}$ to $\mathfrak{W}_{2}$ is said to be limited if there exists a. $C$ such that for all $f \in \mathcal{D}$

$$
\|T f\|_{2} \leqq C\|f\|_{1} \text {. }
$$

The least such $C$ is called the bound of $C$.

THEOREM 1.19. If $T$ is a continuous linear transformation from $\mathfrak{S}_{1}$ to $\mathfrak{S}_{2}$, with bound $C$, then $\tilde{T}$ exists and has the bound $C$ and the domain of $\tilde{T}$ is the closed linear manifold determined by $\mathfrak{D}$.

THEOREM 1.20. If $T$ is a limited transformation with bound $C$ from $\mathfrak{W}_{1}$ to $\mathfrak{W}_{2}$, with domain spanning $\mathfrak{W}_{1}$, then $T^{*}$ exists and is closed and limited with bound $C$ and domain $\mathfrak{W}_{2}$.

THEOREM I. Let $T$ be a closed limited transformation from $\mathfrak{S}_{1}$ to $\mathfrak{S}_{2}$ with bound C. Let $\left\{f_{n}\right\}$ be a sequence everywhere dense in $\mathfrak{D}$. The sequences $\left\{f_{n}, T f_{n}\right\}$ and $\left\{T f_{n}\right\}$ are everywhere dense in $\mathfrak{T}$ and $\Re$ respectively. Let $\left\{\phi_{n}\right\}$ be a sequence which determines $\mathfrak{D}$. Then the sequences $\left\{\phi_{n}, T \phi_{n}\right\}$ and $\left\{T \phi_{n}\right\}$ determine $\mathfrak{T}$ and $\Re$ respectively.

Let $\{f, T f\} \in \mathcal{T}$. Then given an $\epsilon>0$, we can find an $f$ such that

Then

$$
\left\|f_{n}-f\right\|<\frac{\epsilon}{1+C}
$$

$$
\begin{aligned}
\|\{f, & T f\}-\left\{f_{n}, T f_{n}\right\}\left\|_{1,2}=\right\|\left\{f-f_{n}, T\left(f-f_{n}\right)\right\} \|_{1,2} \\
& =\left(\left\|f-f_{n}\right\|_{1}^{2}+\left\|T f-T f_{n}\right\|_{2}^{2}\right)^{1 / 2} \leqq\left\|f-f_{n}\right\|_{1}+\left\|T f-T f_{n}\right\|_{2} \\
& =\left\|f-f_{n}\right\|_{1}+\left\|T\left(f-f_{n}\right)\right\|_{2} \leqq(1+C)\left\|f-f_{n}\right\|_{1}<\epsilon .
\end{aligned}
$$

We have also shown that $\left\|T f-T f_{n}\right\|<\epsilon$.

The proof of the last statement of the theorem is simplified by the following lemma whose proof is almost immediate. It is convenient to introduce the following notation. We denote by $\mathfrak{M}(\subseteq)$ the linear manifold determined by $\mathfrak{S}$, and by $\overline{\mathfrak{M}}(\mathfrak{S})$ the closed linear manifold determined by $\mathfrak{S}$.

LEMMA. A necessary and sufficient condition that a sequence $\left\{k_{n}\right\}$ determine a closed linear manifold $\mathfrak{D}$, is that the denumerable set $R\left(\left\{k_{n}\right\}\right)$ consisting of all elements in the form $\sum_{i}^{n} r_{i} k_{i}, n$ being any integer, the $r_{i}$ being rational complex, be everywhere dense in $\mathfrak{D}$. 
We apply the lemma to show that $R\left(\left\{\phi_{i}\right\}\right)$ is everywhere dense in $\mathfrak{D}$. Then we apply the part of the theorem already proved to show that $R\left(\left\{\left\{\phi_{i}, T \phi_{i}\right\}\right\}\right)$ and $R\left(\left\{T \phi_{i}\right\}\right)$ are everywhere dense in $\mathfrak{T}$ and $\Re$ respectively. These facts and the lemma imply that $\left\{\left\{\phi_{i}, T \phi_{i}\right\}\right\}$ and $\left\{T \phi_{i}\right\}$ determine $\mathfrak{T}$ and $\Re$, respectively.

THEOREM II. Let $T$ be a limited transformation from $\mathfrak{W}_{1}$ to $\mathfrak{W}_{2}$, with domain $\mathfrak{S}_{1}$ and range determining $\mathfrak{S}_{2}$. Then $T^{*}$ exists and is limited with domain $\mathfrak{W}_{2}$ and has an inverse. Let $\left\{\psi_{i}\right\}$ be a complete orthonormal set in $\mathfrak{D}_{1},\left\{\phi_{i}\right\}$ a complete orthonormal set in $\mathfrak{S}_{2}$.

Let $\left\{\chi_{i}\right\}$ be a sequence in $\mathfrak{W}_{1}$, which is obtained by orthonormalizing the sequence $\left\{T^{*} \phi_{i}\right\}$. Then the sequence $\left\{\chi_{i}\right\}$ determines $\mathfrak{W}_{1} \ominus \mathfrak{N}$; or $T f=0$ if and only if $\left(f, \chi_{i}\right)_{1}=0$ for every $i$. A necessary and sufficient condition that an element $g \in \mathfrak{S}_{2}$ be in the range of $T$ is that

$$
\sum_{1}^{\infty}\left|\left(g, T^{*-1} \chi_{i}\right)_{2}\right|^{2}<\infty
$$

If $g \in \Re$ and if

$$
f=\sum_{1}^{\infty}\left(g, T^{*-1} \chi_{i}\right)_{2} \chi_{i}
$$

then $T f=g$.

That $T^{*}$ exists and is limited with domain $\mathfrak{S}_{2}$ is implied by Theorem 1.20. Since the manifold of zeros of $T^{*}, \mathfrak{N}^{*}$, is by Theorem 1.16 equal to $\mathfrak{W}_{2} \ominus \overline{\mathfrak{M}}(\Re), \mathfrak{R}^{*}$ contains only the element 0 , since $\overline{\mathfrak{M}}(\Re)=\mathfrak{S}_{2}$, by hypothesis. Hence $T^{*-1}$ exists.

Since the sequence $\left\{\phi_{i}\right\}$ determines $\mathfrak{W}_{2}$ and $T^{*}$ is limited, it follows from Theorem I that the set $\left\{T^{*} \phi_{i}\right\}$ determines $\overline{\mathfrak{M}}\left(\Re^{*}\right)$ which is $\mathfrak{V}_{1} \ominus \mathfrak{R}$, by Theorem 16. Hence when we orthonormalize the set $\left\{T^{*} \phi_{i}\right\}$, the resulting sequence determines $\mathfrak{W}_{1} \ominus \mathfrak{R}$.

Now one can readily verify that $\left\{\chi_{i}\right\} \subseteq \mathfrak{D}^{*-1}$ and that $\left\{T^{*-1} \chi_{i}\right\}$ determines $\mathfrak{W}_{2}$ since when we orthonormalize $\left\{T^{*-1} \chi_{i}\right\}$ we obtain $\left\{\phi_{i}\right\}$.

Now if $g \in \Re$, there exists an $f^{\prime} \in \mathfrak{S}_{1}$, such that $g=T f^{\prime}$. If $E$ is the projection with range $\overline{\mathfrak{M}}\left(\mathfrak{R}^{*}\right)$, then $I-E$ is the projection with range $\mathfrak{N}$, the manifold of zeros of $T$, by Theorem 1.16. Hence $g=T f^{\prime}=T E f^{\prime}+T(I-E) f^{\prime}=T E f^{\prime}=T f$, letting $E f^{\prime}=f$. Since as shown above the sequence $\left\{\chi_{i}\right\}$ determines $\overline{\mathfrak{M}}\left(\Re^{*}\right)$, we have

where

$$
f=\sum_{1}^{\infty} a_{i} \chi_{i}, \quad \sum_{1}^{\infty}\left|a_{i}\right|^{2}<\infty
$$

$$
a_{i}=\left(f, \chi_{i}\right)_{1}=\left(f, T^{*} T^{*-1} \chi_{i}\right)_{1}=\left(T f, T^{*-1} \chi_{i}\right)_{2}=\left(g, T^{*-1} \chi_{i}\right)_{2} .
$$


Thus the condition stated in the theorem is necessary.

It is also sufficient. For suppose

$$
\sum_{1}^{\infty}\left|a_{i}\right|^{2}<\infty, \quad a_{i}=\left(g, T^{*-1} \chi_{i}\right)_{2} .
$$

Let $f=\sum_{1}^{\infty} a_{i} \chi_{i}$; we will show that $T f=g$. For we have

$$
\left(T^{*-1} \chi_{i}, T \chi_{i}\right)_{2}=\left(T^{*} T^{*-1} \chi_{i}, \chi_{i}\right)_{1}=\left(\chi_{i}, \chi_{i}\right)_{1}=\delta_{i j},
$$

and hence, since $T$ is continuous, for every $i$

$$
\begin{aligned}
\left(g-T f, T^{*-1} \chi_{i}\right)_{2} & =\left(g-\sum_{1}^{\infty} a_{j} T \chi_{j} ; T^{*-1} \chi_{i}\right)_{2} \\
& =\left(g, T^{*-1} \chi_{i}\right)_{2}-\sum_{1}^{\infty} a_{j}\left(T \chi_{i}, T^{*-1} \chi_{i}\right)=\left(g, T^{*-1} \chi_{i}\right)-a_{i} \\
& =\left(g, T^{*-1} \chi_{i}\right)-\left(g, T^{*-1} \chi_{i}\right)=0 .
\end{aligned}
$$

But we have already shown that the sequence $\left\{T^{*-1} \chi_{i}\right\}$ determines $\mathfrak{W}_{2}$. Hence $g-T f=0$ and $g \in \Re$.

If $T$ is not limited and if $\left\{f_{n}\right\}$ is a sequence which determines $\overline{\mathfrak{M}}(\mathfrak{D})$ and each $f_{n} \in \mathfrak{D}$, then the sequence $\left\{f_{n}, T f_{n}\right\}$ does not necessarily determine $\mathfrak{T}$. For instance let $D$ be the transformation in $\mathfrak{\Omega}_{2}$, for the interval $0 \leqq x \leqq 2 \pi$ ((S) Theorem 1.24) with domain consisting of all elements in the form $f=c+\int_{0}^{x} g(\xi) d \xi, g \in \mathfrak{R}_{2}$ and such that $D f=g$. The sequence $\left\{e^{i n} x, n=0, \pm 1\right.$, $\pm 2, \cdots$, determines $\mathfrak{R}_{2}$, but the element $\left\{e^{x}-e^{2 \pi-x}, e^{x}+e^{2 \pi-x}\right\}$ is orthogonal to the sequence $\left\{\left\{e^{i n x}, i n e^{i n x}\right\}\right\}, n=0, \pm 1, \pm 2, \cdots . \dagger$

Note on constructability. It is desirable for the applications which we will make later to have a method of procedure by which one can use Theorem 1.16 and Theorem II without recourse to Zermelo's Axiom. Zermelo's Axiom is used in showing that a sub-set of a separable space is separable. For the applications mentioned it is sufficient to give a method using the operations of the postulates ((S) p. 3) by which having a set which determines $\mathfrak{M}$ we obtain a set which determines $\mathfrak{E} \ominus \mathfrak{M}$. Let $\mathfrak{M}$ be the range of the projection $E$. Let $\left\{f_{i}\right\}$ determine $\mathfrak{M}$. Let $\left\{\chi_{i}\right\}$ be the result of applying the GramSchmidt process to $\left\{f_{i}\right\}$. Let $\left\{\phi_{i}\right\}$ be a complete orthonormal set in $\mathfrak{G}$. Then we notice that

$$
E \phi_{i}=\sum_{1}^{\infty} a_{i} \chi_{i}, \quad a_{i}=\left(\phi_{i}, \chi_{i}\right)
$$

† Compare this example with the results in the paper by J. von Neumann, on non-limited infinite matrices, Journal für Mathematik, vol. 161 (1929), pp. 208-236. It offers a "graphical interpretation" of the general results. 
and if $f \in \mathfrak{Q} \ominus \mathfrak{M}$ then since $E$ is limited

$$
f=(1-E) f=(1-E) \sum_{1}^{\infty} b_{i} \phi_{i}=\sum_{1}^{\infty} b_{i}(1-E) \phi_{i}, \quad b_{i}=\left(f, \phi_{i}\right) .
$$

Hence the sequence $\left\{(1-E) \phi_{i}\right\}$ determines $\mathfrak{E} \Theta \mathfrak{M}$.

\section{ISOMETRIC TRANSFORMATIONS}

DEFINITION 1.14. $U$ will be called a unitary transformation from $\mathfrak{W}_{1}$ to $\mathfrak{W}_{2}$ if its domain is $\mathfrak{W}_{1}$ and its range is $\mathfrak{W}_{2}$ and if for every $f$ and $g$ in its domain we have

$$
(u f, u g)_{2}=(f, g)_{1} \text {. }
$$

Definition 1.15. A linear transformation $V$ from $\mathfrak{W}_{1}$ to $\mathfrak{W}_{2}$ will be said to be isometric if, for every $f$ and $h$ in its domain, we have

$$
(v f, v h)_{2}=(f, h) \text {. }
$$

It is hardly necessary to point out that there is at least one unitary transformation between any two Hilbert spaces, since if $\left\{\phi_{i}\right\}$ is a complete orthonormal set in $\mathfrak{S}_{1}$ and if $\left\{\psi_{i}\right\}$ is a complete orthonormal set in $\mathfrak{W}_{2}$ and if $\mathfrak{T}=\left\{\left\{\phi_{i}, \psi_{i}\right\}\right\}$, then $U=\tilde{T}$ is obviously unitary.

The proofs of the following two theorems are quite similar to the proofs of (S) Theorems 2.42 and 2.43.

THEOREM 1.21. A unitary transformation $U$ from $\mathfrak{W}_{1}$ to $\mathfrak{W}_{2}$ is linear continuous and isometric. $U^{*}$ exists and is unitary. $U^{-1}$ exists and $U^{-1}=U^{*}$.

THEOREM 1.22. An isometric transformation $V$ from $\mathfrak{W}_{1}$ to $\mathfrak{W}_{2}$ is limited and possesses an isometric inverse. The transformation $\tilde{V}$ exists and is isometric. The domain and range of $\tilde{V}$ are the closed linear manifolds determined by the domain and range of $V$ respectively.

\section{VON NEUMANN'S THEOREM}

By means of the previous discussion, we are now able to obtain results corresponding to the remaining Theorems in $(\mathrm{N})$ in a manner analogous to the proofs given in that memoir. These results follow.

We introduce the transformation $B=\left(T^{*} T\right)^{1 / 2}$, which is of course in $\mathfrak{W}_{1}$.

THEOREM 1.23. If $T$ is a closed linear transformation from $\mathfrak{S}_{1}$ to $\mathfrak{W}_{2}$, with domain everywhere dense in $\mathfrak{S}_{1}$, and if $B=\left(T^{*} T\right)^{1 / 2}, B$ is self-adjoint in $\mathfrak{S}_{1}$, with domain $\mathfrak{D}$ and $(T f, T g)_{2}=(B f, B g)_{1}$ for all $f$ and $g$ of $\mathfrak{D}$. 
THEOREM 1.24. Let $T$ be a closed linear transformation from $\mathfrak{W}_{1}$ to $\mathfrak{W}_{2}$, with domain everywhere dense in $\mathfrak{W}_{1}$. Let $B=\left(T^{*} T\right)^{1 / 2}, C=\left(T T^{*}\right)^{1 / 2} ; B$ and $C$ are self-adjoint. The domain of $B$ is $\mathfrak{D}$, the domain of $C$ is $\mathfrak{D}^{*}$. Let $\Re_{b}$ denote the range of $B, \Re_{c}$ that of $C$. Let $\mathfrak{R}$ and $\mathfrak{R}^{*}$ be as in Theorem 1.16. $B f=0$ if and only if $f \in \mathfrak{R}, C f=0$ if and only if $f \in \mathfrak{N}^{*}$. Also

$$
\overline{\mathfrak{M}}\left(\Re_{b}\right)=\overline{\mathfrak{M}}\left(\Re^{*}\right)=\mathfrak{W}_{1} \ominus \mathfrak{N} ; \overline{\mathfrak{M}}(\Re)=\overline{\mathfrak{M}}\left(\Re_{c}\right)=\mathfrak{W}_{2} \ominus \mathfrak{N}^{*} .
$$

Let $E$ be the projection in $\mathfrak{W}_{1}$ on $\mathfrak{S}_{1} \ominus \mathfrak{R}=\overline{\mathfrak{M}}\left(\Re^{*}\right)$, $E^{\prime}$ be the projection in $\mathfrak{W}_{2}$ on $\overline{\mathfrak{M}}(\Re)$.

There is a transformation $W$ from $\mathfrak{W}_{1}$ to $\mathfrak{W}_{2}$, with domain $\mathfrak{W}_{1}$ and continuous, which takes $\overline{\mathfrak{M}}\left(\Re_{b}\right)$ in a single-valued isometric manner onto $\overline{\mathfrak{M}}(\Re) ; W^{*}$ takes $\overline{\mathfrak{M}}\left(\Re_{c}\right)$ in a single-valued isometric manner onto $\overline{\mathfrak{M}}\left(\Re^{*}\right)$. W is zero on $\mathfrak{\Re}, W^{*}$ is zero on $\mathfrak{R}^{*}$.

Furthermore $W^{*} W=E, W W^{*}=E^{\prime}$,

$$
\begin{aligned}
T & =W B=C W ; & T^{*} & =B W^{*}=W^{*} C ; \\
B & =W^{*} T=T^{*} W ; & C & =T W^{*}=W T^{*} ; \\
C & =W B W^{*} ; & B & =W^{*} C W .
\end{aligned}
$$

The following theorem is now a simple consequence of (S) Theorem 2.25.

THEOREM 1.25. If $T$ is a closed transformation from $\mathfrak{W}_{1}$ to $\mathfrak{W}_{2}$ with domain $\mathfrak{S}_{1}$, then $T$ is bounded. $\dagger$

\section{BREAKAge}

A closed linear transformation is a correspondence which is isomorphic with respect to Postulate $A,(S)$ p. 3. We will study in this section to what extent the relationship of orthogonality, introduced in Postulate $\mathrm{B}$, is preserved.

DeFINITION I. Let $T$ be a closed linear transformation from $\mathfrak{W}_{1}$ to $\mathfrak{W}_{2}$, with domain everywhere dense in $\mathfrak{W}_{1}$. A projection $F$ in $\mathfrak{S}_{1}$ is said to break $T$ if

(1) $F f \in \mathfrak{D}$ for all $f \in \mathfrak{D}$, and

(2) there exists a projection $F^{\prime}$ (independent of $f$ ) such that $T F f=F^{\prime} T f$ for all $f \in \mathfrak{D}$.

If $F$ breaks $T$ and $\mathfrak{M}$ is the range of $F$, then $\mathfrak{M}$ will be said to break $T$.

We note that conditions (1) and (2) are equivalent to $T F \supseteq F^{\prime} T$.

THEOREM III. If $F$ breaks $T$, a closed linear transformation from $\mathfrak{W}_{1}$ to $\mathfrak{S}_{2}$ with domain everywhere dense in $\mathfrak{S}_{1}$, then $F^{\prime}$ breaks $T^{*}$ and $T^{*} F^{\prime} f=F T^{*} f$ for all $f \in \mathfrak{D}^{*}$.

† (B) chap. III, Theorem 7, p. 41. 
For all $f \in \mathscr{D}$ and $g \in \mathfrak{D}^{*}$, we have

$$
\left(T f, F^{\prime} g\right)_{\mathbf{2}}=\left(F^{\prime} T f, g\right)_{2}=(T F f, g)_{2}=\left(F f, T^{*} g\right)_{1}=\left(f, F T^{*} g\right) .
$$

But this equation implies $T^{*} F^{\prime} g=F T^{*} g$ for all $g \in \mathfrak{D}^{*}$, or $T^{*} F^{\prime} \supseteq F T^{*}$.

Henceforth all quantities will have their significance as in Theorem 1.24.

THEOREM IV. $F$ breaks $T$, if and only if it reduces $B$. If $F$ breaks $T$, then $F^{\prime}=W F W^{*}+F_{0}^{\prime}$, where $F_{0}^{\prime}$ is a projection whose range is included in $\mathfrak{R}^{*}=\mathfrak{G}_{2} \ominus \overline{\mathfrak{M}}(\Re)$ and for any such $F^{\prime}, T F \supseteq F^{\prime} T$.

We first show that if $F$ reduces $B$, then $W F W^{*}$ is a projection. $W F W^{*}$ is obviously a limited symmetric transformation. We must show that $\left(W F W^{*}\right)^{2}$ $=W F W^{*}$. To do this we prove that $E F W^{*}=F W^{*}$. Let $W^{* \prime}$ be the contraction of $W^{*}$ with domain $\Re \cdot+\cdot \Re^{*}$. Since the domain of $W^{* \prime}$ and also $W^{*}$ are linear, $W^{* \prime}$ is linear and also limited since $W^{*}$ is limited.

Since, by hypothesis, $F$ reduces $B$, and by Theorem $1.24, W^{*} T=B=T^{*} W$,

$$
F W^{* \prime} T=F W^{*} T=F B \subseteq B F=T^{*} W F .
$$

Thus, since $E$ is the projection on $\bar{\Re}(\Re)$, we have

$$
E F W^{* \prime} T=F W^{* \prime} T,
$$

or $E F W^{* \prime} f=F W^{* \prime} f, f \in \Re$. But for $g \epsilon \Re^{*}, W^{* \prime} g=W^{*} g=0$ and $E F W^{* \prime} g$ $=F W^{* \prime} g=0$. Now an element of $\Re \cdot+\cdot \Re^{*}$ is the sum of an element of $\Re$ and an element of $\mathfrak{R}^{*}$, and we can conclude

$$
E F W^{* \prime}=F W^{* \prime} \text {. }
$$

But $\Re \cdot+\cdot \mathfrak{N}^{*}$, the domain of this transformation, is everywhere dense in $\mathfrak{S}_{2}$. Hence by Theorem 1.19 , it has a unique closed extension and thus $E F W^{*}=F W^{*}$, since both are closed and $E F W^{*} \supseteq E F W^{* \prime}$ and $F W^{*} \supseteq F W^{* \prime}$.

Now

$$
\begin{aligned}
\left(W F W^{*}\right)^{2} & =\left(W F W^{*}\right)\left(W F W^{*}\right)=(W F)\left(W^{*} W\right)\left(F W^{*}\right)=(W F)(E)\left(F W^{*}\right) \\
& =(W F)\left(E F W^{*}\right)=(W F)\left(F W^{*}\right)=(W)(F F)\left(W^{*}\right)=W F W^{*} .
\end{aligned}
$$

Hence by (S) Theorem 2.36, $W F W^{*}$ is a projection. Now since the domain of $B$ is the same as that of $T, F f \in \mathfrak{D}$ if $f \in \mathfrak{D}$. We also have, since $B=W^{*} T$ and $E^{\prime}=W W^{*}$ is the projection on the closed linear manifold determined by $\Re$,

$$
T F=E^{\prime} T F=(W)\left(W^{*} T F\right)=W B F \supseteq W F B=W F W^{*} T .
$$

Now conversely let us suppose that $F$ breaks $T$ and let $F^{\prime}$ be as in Definition I. Then by Theorem III, we have

$\dagger \Re \cdot+\cdot \Re^{*}$ contains every element which is the sum of an element of $\Re$ and an element of $\Re^{*}$ and only such elements. 


$$
F T^{*} T \subseteq T^{*} F^{\prime} T \subseteq T^{*} T F .
$$

One infers from this that $F$ reduces $T^{*} T=B B$ and hence, by a simple application of (S) Theorem 8.1, that $F$ reduces $B$.

Let $F$ break $T$. Now suppose $F^{\prime}$ is such that $T F \supseteq F^{\prime} T$. Then $F^{\prime}$ is in the form stated in the theorem. For we have for all $f \in \mathfrak{D}$,

$$
T F f=W F W^{*} T f=F^{\prime} T f .
$$

Hence $W F W^{*} f=F^{\prime} f, f \in \Re$.

Now let $H$ be the contraction of $W F W^{*}$, with domain $\Re$. Since $\Re$ and $W F W^{*}$ are linear, $H$ is linear. Let $K$ be the contraction of $F^{\prime}$, with domain $\Re$. It is also linear, and from the above we have that $H=K$. Applying Theorem 1.19 , we have

$$
\tilde{H}=\tilde{K},
$$

and this transformation has domain $\overline{\mathfrak{M}}(\Re)$. It is also a contraction of $W F W^{*}$ and $F^{\prime}$. Hence since the range of $E^{\prime}$ is $\overline{\mathfrak{M}}(\Re)$,

$$
W F W^{*} E^{\prime}=F^{\prime} E^{\prime} \text {. }
$$

But it follows from Theorem 1.24 that $W^{*} E^{\prime}=W^{*}$. Hence

$$
W F W^{*}=F^{\prime} E^{\prime} \text {. }
$$

But (S) Theorem 2.37 now yields, since $W F W^{*}$ is a projection as shown above, $F^{\prime} E^{\prime}=E^{\prime} F^{\prime}$ and hence $W F W^{*}=E^{\prime} F^{\prime}$ and

$$
(I-E) F^{\prime}=F^{\prime}-E^{\prime} F^{\prime}=F^{\prime}-F^{\prime} E^{\prime}=F^{\prime}\left(I-E^{\prime}\right) .
$$

Hence by (S) Theorem 2.37, $\left(I-E^{\prime}\right) F^{\prime}$ is a projection and its range is included in $\mathfrak{W}_{2} \ominus \overline{\mathfrak{M}}(\Re)=\mathfrak{R}^{*}$. Let $F_{0}^{\prime}=\left(I-E^{\prime}\right) F^{\prime} ;$ then

$$
F^{\prime}=E^{\prime} F^{\prime}+\left(I-E^{\prime}\right) F^{\prime}=W F W^{*}+F_{0}^{\prime} .
$$

Conversely, since the range of $W F W^{*}$ is included in the range of $W$, $\overline{\mathfrak{M}}(\Re)$, if $F_{0}^{\prime}$ is a projection, whose range is included in $\mathfrak{W}_{2} \ominus \overline{\mathfrak{M}}(\Re)=\mathfrak{N}^{*}$, then

$$
F^{\prime}=W F W^{*}+F_{0}^{\prime}
$$

is a projection by (S) Theorem 2.37 and

$$
T F \supseteq W F W^{*} T=\left(W F W^{*}+F_{0}{ }^{\prime}\right) T=F^{\prime} T .
$$

We have thus shown that if $F$ breaks $T, F^{\prime}$ must be in the form stated and any such $F^{\prime}$ will satisfy the definition of breakage.

'THEOREM V. If $T$ is a closed linear transformation in $\mathfrak{S}$, with domain everywhere dense in $\mathfrak{b}$, then $F$ reduces $T$ if and only if it reduces $B$ and $W$. 
Now if $F$ reduces $T$, it breaks $T$, since we may take $F^{\prime}=F$, and hence it reduces $B$ and we obtain by the previous theorem

$$
F=F^{\prime}=W F W^{*}+F_{0}{ }^{\prime},
$$

where $F_{0}^{\prime}$ is a projection, whose range is included in $\mathfrak{R}^{*}$, and hence $W^{*} F^{\prime}=0$. Also in the proof of the previous theorem we have shown that $E F W^{*}=F W^{*}$ and so we obtain

$$
W^{*} F=W^{*}\left(W F W^{*}+F_{0}{ }^{\prime}\right)=W^{*} W F W^{*}+W^{*} F_{0}{ }^{\prime}=E F W^{*}+0=F W^{*} .
$$

Since the domain of $W^{*}$ is $\mathfrak{E}$, this yields that $F$ reduces $W^{*}$.

But if $F$ reduces $W^{*}$, it reduces $W$, since the equation $F W^{*}=W^{*} F$ implies by taking adjoints $W^{* *} F=F W^{* *}$ or $W F=F W$.

On the other hand if $F$ reduces both $B$ and $W$, it reduces $T$. For since the domain of $B$ is $\mathfrak{D}$, the domain of $T$, and since $F$ reduces $B, F f \in \mathfrak{D}$ for all $f \in \mathfrak{D}$ and

$$
T F f=W B F f=W F B f=F W B f=F T f .
$$

THEOREM VI. Let $T$ be as in Theorem 1.24. Then there exists a set of linear transformations $\left\{T_{i}\right\}, i=0, \pm 1, \pm 2, \cdots$, each closed and limited with a limited inverse, with domain $\mathfrak{D}_{i}$ and range $\Re_{i}$, such that $\mathfrak{D}_{i} \perp \mathfrak{D}_{j}$ and $\Re_{i} \perp \Re_{j}$ for for $i \neq j$ and such that for all $f \in \mathfrak{W}_{1}$,

$$
f=F f+\sum_{-\infty}^{\infty} f_{i}, \quad f_{i} \in \mathfrak{D}_{i}, \quad T f=\sum_{-\infty}^{\infty} T_{i} f_{i}, \dagger
$$

where $F$ is the projection on the manifold of zeros of $T . \ddagger$

We take any set of numbers $\left\{\alpha_{i}\right\}$ such that $i=0, \pm 1, \pm 2, \cdots, \alpha_{i}>\alpha_{i}$ if $i>j, \lim _{i \rightarrow-\infty} \alpha_{i}=0, \lim _{i \rightarrow \infty} \alpha_{i}=\infty$. We let $F_{i}=E\left(\alpha_{i}\right)-E\left(\alpha_{i-1}\right)$, where $E(\lambda)$ is the resolution of the identity corresponding to $B$. Now it is shown in (S) Theorem 5.9 that the range of $F_{i}$ is in the domain of $B$ which is $\mathfrak{D}$, the domain of $T$. Hence we may take $T_{i}$ as the contraction $T$, with domain the range of $F_{i}$.

It is also shown in the proof of (S) Theorem 5.7 that $F_{i}$ is a projection and that the ranges of $F_{i}$ and $F_{j}$ for $i \neq j$ are mutually orthogonal or that $\mathfrak{D}_{i} \perp \mathfrak{D}_{j}$. In the proof of (S) Theorem 5.9 we learn that

$$
\left\|B F_{i}\right\|^{2}=\int_{\alpha_{i-1}}^{\alpha_{i}} \lambda^{2} d\|E(\lambda) f\|^{2} .
$$

$\dagger$ This equation is also to imply that $T f$ has a meaning if and only if the expression on the right has a meaning.

$\ddagger$ Theorem VI compensates in the study of the inverses of contractions of $T$ for the lack of compactness of Hilbert space. In treating certain types of partial differential equations, it has been possible to use spaces of the Banach type having a species of compactness. Cf. J. Schauder, Mathematische Annalen, vol. 106, pp. 661-721. 
Hence we have that

$$
\alpha_{i-1}^{2}\left(\left\|E\left(\alpha_{i}\right) f\right\|^{2}-\left\|E\left(\alpha_{i-1}\right) f\right\|^{2}\right) \leqq\left\|B F_{i}\right\|^{2} \leqq \alpha_{i}^{2}\left(\left\|E\left(\alpha_{i}\right) f\right\|^{2}-\left\|E\left(\alpha_{i-1}\right) f\right\|^{2}\right) .
$$

Now if $f$ is in the range of $F_{i}$, it is by (S) Theorem 2.37 perpendicular to the range of $E\left(\alpha_{i-1}\right)$ and furthermore $E\left(\alpha_{i}\right) f=f$. Hence for all $f \in \mathfrak{D}_{i}$,

$$
\alpha_{i-1}^{2}\|f\|^{2} \leqq\left\|B_{i} f\right\|^{2} \leqq \alpha_{i}^{2}\|f\|^{2} .
$$

Since for $f \in \mathfrak{D}_{i}$,

$$
\left\|T_{i} f\right\|=\|T f\|=\|B f\|=\left\|B_{i} f\right\|,
$$

(2) yields that $T_{i}$ is limited with a limited inverse, and since its domain is a closed linear manifold, it is closed.

Now (S) Theorem 5.8 tells us that $\mathfrak{D}_{i}$ reduces $B$ and hence by Theorem IV we have that $F_{i}$ breaks $T$.

We will show that $\Re_{i} \perp \Re_{j}, i \neq j$. For let $T_{i} f \in \Re_{i}$ and $T_{j} g \in \Re_{j}$. Hence $F_{i} f=f$, $F_{j} g=g$. Now since $T$ and $B$ are isometrically equivalent, and $F_{i}$ and $F_{j}$ reduce $B$,

$$
\left(T_{i} f, T_{j} g\right)_{2}=\left(B_{i} f, B_{j} g\right)_{1}=\left(B_{i} F_{i} f, B_{j} F_{j} g\right)_{1}=\left(F_{i} B_{i} f, F_{j} B_{j} g\right)=0,
$$

since the ranges of $F_{i}$ and $F_{j}$ are mutually orthogonal.

By Definition 5.1 (S),

$F+\sum_{-\infty}^{\infty} F_{i}=F+\lim _{\alpha_{n} \rightarrow \infty, \alpha_{-m} \rightarrow 0}\left(E\left(\alpha_{n}\right)-E\left(\alpha_{-m}\right)\right)=F+I-E(0)=I-E(0-)$,

remembering that $F$ is the manifold of zeros of $B$. Since $B$ is not negative definite ((S) Definition 2.14), (S) Theorem 5.12 yields that $E(0-)=0$. Hence

$$
F+\sum_{-\infty}^{\infty} F_{i}=I
$$

Hence for any $f \in \mathfrak{W}_{1}$,

letting $F_{i} f=f_{i}, f_{i} \in \mathfrak{D}_{i}$.

$$
f=F f+\sum_{-\infty}^{\infty} F_{i} f=F f+\sum_{-\infty}^{\infty} f_{i}
$$

Since $B F f=0, B$ is linear and $\lim _{i \rightarrow \infty} \alpha_{i}=0$,

$$
\begin{aligned}
\sum_{-\infty}^{N} B_{i} f_{i} & =\sum_{-\infty}^{N} B\left(E\left(\alpha_{i}\right)-E\left(\alpha_{i-1}\right)\right) f=B E\left(\alpha_{n}\right) f-\lim _{i \rightarrow-\infty} B E\left(\alpha_{i}\right) f \\
& =B E\left(\alpha_{n}\right) f-\lim _{\alpha_{i} \rightarrow 0} B E\left(\alpha_{i}\right) f=B E\left(\alpha_{n}\right) f-\lim _{\alpha_{i} \rightarrow 0} B\left(E\left(\alpha_{i}\right)-F\right) f .
\end{aligned}
$$

Since $B$ is not negative definite, (S) Theorem 5.12 yields $F=E(0)$, and by Theorem 5.9 (S), 
Hence

$$
\left\|B\left(E\left(\alpha_{i}\right)-E(0)\right) f\right\|^{2}=\int_{0}^{\alpha_{i}} \lambda^{2} d\|E(\lambda) f\|^{2} \leqq \alpha_{i}^{2}\|f\|^{2} .
$$

$$
\lim _{\alpha_{i} \rightarrow 0} B\left(E\left(\alpha_{i}\right)-F\right) f=\lim _{\alpha_{i} \rightarrow 0} B\left(E\left(\alpha_{i}\right)-E(0)\right) f=0,
$$

and from (3) we get

$$
\sum_{-\infty}^{N} B_{i} f_{i}=B\left(E\left(\alpha_{n}\right) f\right) .
$$

Since $B$ is not negative definite, (S) Theorem 5.12 yields $E(-N)=0$ for $N>0$. Let $\Delta_{N}=\left(-N, \alpha_{N}\right)$; then

$$
\begin{aligned}
\sum_{-\infty}^{\infty} B_{i} f_{i} & =\lim _{N \rightarrow \infty} \sum_{-\infty}^{N} B_{i} f_{i}=\lim _{N \rightarrow \infty}\left(\sum_{-\infty}^{N} B_{i} f_{i}\right)-\lim _{N \rightarrow \infty} B E(-N) f \\
& =\lim _{N \rightarrow \infty} B\left(E\left(\alpha_{n}\right)-E(-N)\right) f=\lim _{N \rightarrow \infty} B E\left(\Delta_{N}\right) f .
\end{aligned}
$$

Now $\lim _{n \rightarrow \infty} \alpha_{n}=\infty$, and in the proof of (S) Theorem 5.9, it is shown that the limit on the right exists if and only if $B f$ exists, and if $B f$ exists, then this limit equals $B f$. Hence

$$
B f=\sum_{-\infty}^{\infty} B_{i} f_{i}:
$$

Now since $W$ is isometric on $\overline{\mathfrak{M}}\left(\Re_{b}\right)$,

$$
\sum_{-\infty}^{\infty} W B_{i} f_{i}
$$

converges if and only if $\sum_{-\infty}^{\infty} B_{i} f_{i}$ converges, and hence since the domain of $T$ is the same as the domain of $B$ if and only if $f \in \mathfrak{D}$, and when $f \in \mathfrak{D}$, we have

since $W$ is continuous.

$$
T f=W B f=W \sum_{-\infty}^{\infty} B_{i} f_{i}=\sum_{-\infty}^{\infty} W B_{i} f_{i}=\sum_{-\infty}^{\infty} T_{i} f_{i},
$$

THEOREM VII. Let $T$ be a closed and linear transformation from $\mathfrak{b}_{1}$ to $\mathfrak{W}_{2}$, with domain everywhere dense in $\mathfrak{W}_{1} . T^{*}$ exists and is a closed linear transformation from $\mathfrak{W}_{2}$ to $\mathfrak{W}_{1}$, with domain everywhere dense in $\mathfrak{S}_{2}$ (Theorem 1.13). If $\mathfrak{M}_{1} \in \mathfrak{W}_{1}$ and $\mathfrak{M}_{2} \in \mathfrak{S}_{2}$ are the ranges of two projections $F_{1}$ and $F_{2}$ and are such that

(1) if $f \in \mathfrak{D}$, then $F_{1} f \in \mathfrak{D}$;

(2) if $f \in \mathfrak{D}^{*}$, then $F_{2} f \in \mathfrak{D}^{*}$;

(3) if $f \in \mathfrak{M}_{1} \cdot \mathfrak{D}$, then $T f \in \mathfrak{M}_{2}$;

(4) if $f \in \mathfrak{M}_{2} \cdot \mathfrak{D}^{*}$, then $T^{*} f \in \mathfrak{M}_{1}$;

then $F_{1}$ breaks $T$ and $T F_{1} f=F_{2} T f$ for all $f \in \mathcal{D}$. 
Due to condition (1), if we can prove that $T F_{1} f=F_{2} T f$ for every $f \in \mathfrak{D}$, the theorem follows. From condition (3) we notice that for all $f \in \mathfrak{D}$,

$$
F_{2} T F_{1} f=T F_{1} f \text {. }
$$

Now if $f \in \mathfrak{D}$, and $g \in \mathfrak{D}^{*}$, we have by condition (2)

$$
\begin{aligned}
\left(T F_{1} f-F_{2} T f, g\right)_{2} & =\left(F_{2} T F_{1} f-F_{2} T f, g\right)_{2}=\left(T F_{1} f-T f, F_{2} g\right)_{2} \\
& =\left(F_{1} f-f, T^{*} F_{2} g\right)_{1} .
\end{aligned}
$$

But $F_{1} f-f \in \mathfrak{S}_{1} \ominus \mathfrak{M}_{1}$, while by (4) $T^{*} F g \in \mathfrak{M}_{1}$. Hence for all $f \in \mathfrak{D}, g \in \mathfrak{D}^{*}$,

$$
\left(\left(T F_{1}-F_{1} T\right) f, g\right)_{2}=0 .
$$

But $\mathfrak{D}^{*}$ is everywhere dense in $\mathfrak{S}_{2}$, hence for every $f \in \mathfrak{D}$,

$$
T F_{1} f=F_{2} T f .
$$

\section{Functions of CLASS $H$}

Let $S$ be a bounded connected open region. Let $S_{x}$ denote the projection of $S$ on the $x$-axis, $S_{y}$ denote the projection of $S$ on the $y$-axis. We shall use the symbol $m_{1}(E)$ to denote the linear measure of a linear set.

Definition II. A function $f=f(x, y)$ shall be said to be of class $H$ on $S$ (symbolically $f_{\epsilon} H_{s}$ ), if it satisfies the following conditions:

1. It has a summable square and has almost everywhere first and second partial derivatives of summable square on the region $S$.

2.a. There exists a set $\Gamma_{y}$ on the $y$-axis, such that $\Gamma_{y} \subseteq S_{y}, m_{1}\left(S_{y}-\Gamma_{y}\right)=0$ and if $y \in \Gamma_{y}$, then $f(x, y), f_{x}(x, y)$ and $f_{y}(x, y)$ are absolutely continuous functions of $x$, defined for all $x$ such that $(x, y) \epsilon S$.

2.b. There exists a set $\Gamma_{x}$ on the $x$-axis, such that $\Gamma_{x} \subseteq S_{x}, m_{1}\left(S_{x}-\Gamma_{x}\right)=0$, and if $x \in \Gamma_{x}$, then $f(x, y), f_{x}(x, y)$ and $f_{y}(x, y)$ are absolutely continuous functions of $y$ defined for all $y$ such that $(x, y) \epsilon S$.

For $f$ and $g \in H_{s}$, let

$$
\begin{aligned}
\|f-g\|^{2}= & \iint_{S}\left(|f-g|^{2}+\left|f_{x}-g_{x}\right|^{2}+\left|f_{y}-g_{y}\right|^{2}+\left|f_{x x}-g_{x x}\right|^{2}\right. \\
& \left.+\left|f_{x y}-g_{x y}\right|^{2}+\left|f_{y x}-g_{y x}\right|^{2}+\left|f_{y y}+g_{y y}\right|^{2}\right) d S .
\end{aligned}
$$

LEMMA. Given a sequence of functions $\left\{f^{(n)}\right\}, f^{(n)} \in H_{\text {, }}$, such that

$$
\lim _{n+m \rightarrow \infty}\left\|f^{(n)}-f^{(m)}\right\|=0 .
$$

Then there exists an $f_{\epsilon} H_{s}$, such that

$$
\lim _{n \rightarrow \infty}\left\|f-f^{(n)}\right\|=0 .
$$


The sequence $\left\{f_{x, x}^{(n)}\right\}$ is convergent in the mean, hence there exists, by the Riesz-Fischer Theorem, a function $f_{1,1}$ of summable square such that

$$
\lim _{n \rightarrow \infty} \iint_{S}\left|f_{x, x}^{(n)}-f_{1,1}\right|^{2} d S=0 .
$$

But by Fubini's Theorem, we have that

$$
\iint_{S}\left|f_{x, x}^{(n)}-f_{1,1}\right|^{2} d S=\int_{S_{y}} d y \int_{S_{1}(y)}\left|f_{x, x}^{(n)}-f_{1,1}\right|^{2} d x
$$

where $S_{1}(y)$ denotes the set of points in $S$ whose ordinate is $y$. Nowif we set

$$
\phi_{n}^{2}(y)=\int_{S(y)}\left|f_{x, x}^{(n)}(x, y)-f_{1,1}(x, y)\right|^{2} d x,
$$

we see from the above that

$$
\lim _{n \rightarrow \infty} \int_{S_{y}}\left|\phi_{n}(y)-0\right|^{2} d y=0 .
$$

Hence by the Riesz-Fischer Theorem, there exists a subsequence $\left\{\psi_{n}\right\}$ of $\left\{\phi_{n}\right\}$ (and a corresponding subsequence $\left\{g^{(n)}\right\}$ of the sequence $\left\{f^{(n)}\right\}$ ), and a linear set $C_{x, x}^{\prime}, C_{x, x}^{\prime} \subseteq S_{y}$ for which $m_{1}\left(S_{y}-C_{x x}^{\prime}\right)=0$, such that if $y \in C_{x, x}^{\prime}$, then

$$
0=\lim _{n \rightarrow \infty} \psi_{n}^{2}(y)=\lim _{n \rightarrow \infty} \int_{S(y)}\left|g_{x, x}^{(n)}(x, y)-f_{1,1}(x, y)\right|^{2} d x .
$$

Now the sequence $\left\{g_{x, y}^{(n)}\right\}$ converges in the mean also, to some function of summable square $f_{1,2}$, and in a similar manner, we can find a subsequence $\left\{h^{(n)}\right\}$ of the sequence $\left\{g^{(n)}\right\}$ and a set $C_{x, y}^{\prime} \subseteq S_{x}, m_{1}\left(S_{x}-C^{\prime}{ }_{, y}\right)=0$, such that for $x \in C_{x, y}^{\prime}$,

$$
\lim _{n \rightarrow \infty} \int_{S_{2}(x)}\left|h_{x, y}^{(n)}(x, y)-f_{1,2}(x, y)\right|^{2} d y=0 .
$$

The sequence $\left\{h_{x}^{(n)}\right\}$ converges in the mean. Hence by the RieszFischer Theorem, there exists a subsequence $\left\{k_{x}^{(n)}\right\}$, a function $f_{1}$, and a set $S^{\prime} \subseteq S, m S^{\prime}=m S$, such that on $S^{\prime}$

$$
\lim _{n \rightarrow \infty} k_{x}^{(n)}=f_{1} .
$$

Now let $\Gamma_{y}{ }^{(n)}$ correspond to $f^{(n)}$ as $\Gamma_{y}$ did to $f$ in the definition of a function of class $H$ on $S$. Let $C_{x, x}$ denote $C_{x, x} \Pi \Gamma_{y}{ }^{(n)}$. One can readily verify that 
$m_{1}\left(S_{y}-C_{x, x}\right)=0$. Similarly, we define $C_{x, x}=C_{x, y}^{\prime} \Pi \Gamma_{x}^{(n)}$ and obtain

$$
m_{1}\left(S_{x}-C_{x, y}\right)=0 .
$$

Now since $m\left(S^{\prime}\left(C_{x, y} \times C_{x, x}\right)\right)=m S \neq 0$, there is a point $P:(a, b) \epsilon S^{\prime}\left(C_{x, y}\right.$ $\left.\times C_{x, x}\right)$. Let $S_{1}$ consist of all points $(x, y) \epsilon S$ such that either $y \epsilon C_{x, x}$ or $x \in C_{x, y}$. Let $Q:\left(x_{0}, y_{0}\right) \epsilon S_{1}$. Then we can connect $P$ and $Q$ by a polygonal line $\Gamma$, $P R_{1} R_{2} \cdots R_{n} Q$, such that each segment $P R_{1} \subset S_{1}, R_{i} R_{i+1} \subset S_{1}, R_{n} Q \subset S_{1}$ and each segment is parallel to an axis.

Now if a segment of $\Gamma$ is parallel to the $x$-axis, it follows from (1) that $f_{1,1}$ is measurable (linearly) along the segment and has a summable square, by the Riesz-Fischer Theorem. Hence $f_{1,1}$ is also summable since for a finite interval the summability of the square implies the summability of the function. Similarly along a segment of $\Gamma$ parallel to the $y$-axis, $f_{1,2}$ is summable by (2). Thus we may define

$$
f_{x}(Q)=f_{1}(P)+\int_{\Gamma} f_{1,1} d x+f_{1,2} d y .
$$

We now show that the definition of $f_{x}(Q)$ is independent of the choice of $\Gamma$ or of $P$, in fact that $f_{x}(Q)=f_{1}(Q)$, whenever the former is defined.

For, suppose $R_{i} R_{i+1}$ is a segment of $\Gamma$, parallel to the $x$-axis, i.e., $R_{i}$ is $\left(x_{1}, y\right), R_{i+1}$ is $\left(x_{2}, y\right), y \in C_{x, x}$. Since $C_{x, x} \supseteq \Gamma_{x}^{(n)}, f_{x, y}(x, y)$ is absolutely continuous, and

$$
\int_{x_{2}}^{x_{1}} f_{x, x}^{(n)}(\xi, y) d \xi=f_{x}^{(n)}\left(x_{1}, y\right)-f_{x}^{(n)}\left(x_{2}, y\right),
$$

and by Schwarz's Inequality (remembering that $\left\{k^{(n)}\right\}$ is a subset of $\left\{f^{(n)}\right\}$ ),

$$
\begin{aligned}
\left|k_{x}^{(n)}\left(x_{1}, y\right)-f_{x}\left(x_{1}, y\right)-\left(k_{x}^{(n)}\left(x_{2}, y\right)-f_{x}\left(x_{2}, y\right)\right)\right|^{2} \\
\quad=\left|\int_{x_{2}}^{x_{1}}\left(k_{x}^{(n)}(\xi, y)-f_{1,1}(\xi, y)\right) d \xi\right|^{2} \\
\quad \leqq\left|x_{1}-x_{2}\right|\left|\int_{x_{2}}^{x_{1}}\right| k_{x, x}^{(n)}(\xi, y)-\left.f_{1,1}(\xi, y)\right|^{2} d \xi \mid .
\end{aligned}
$$

Since $\left\{k^{(n)}\right\} \subseteq\left\{g^{(n)}\right\}$, it follows from (1) that

(6) $\quad \lim _{n \rightarrow \infty}\left|\left(k_{x}^{(n)}\left(x_{1}, y\right)-f_{x}\left(x_{1}, y\right)\right)-\left(k_{x}^{n)}\left(x_{2}, y\right)-f_{x}\left(x_{2}, y\right)\right)\right|^{2}=0$.

If, on the other hand, $R_{j} R_{j+1}$ is parallel to the $y$-axis and if $R_{j}=\left(x, y_{1}\right)$, $R_{j+1}=\left(x, y_{2}\right), x \in C_{x, y}$, we obtain similarly

$$
\lim _{n \rightarrow \infty}\left|\left(k_{x}^{(n)}\left(x, y_{1}\right)-f_{x}\left(x, y_{1}\right)\right)-\left(k_{x}^{(n)}\left(x, y_{2}\right)-f_{x}\left(x, y_{2}\right)\right)\right|^{2}=0 .
$$


Now either (6) or (7) applies to $P R_{1}$. We also have by the choice of $P:(a, b)$ $\lim _{n \rightarrow \infty} k_{x}^{(n)}(a, b)=f_{x}(a, b)$.

Hence

$$
\lim _{n \rightarrow \infty} k_{x}^{(n)}\left(R_{1}\right)=f_{x}\left(R_{1}\right) .
$$

Similarly, we may continue to apply either (6) or (7), obtaining

$$
\lim _{n \rightarrow \infty} k_{x}^{(n)}\left(R_{i}\right)=f_{x}\left(R_{i}\right), \quad \lim _{n \rightarrow \infty} k_{x}^{(n)}(Q)=f_{x}(Q) .
$$

But the limit of the sequence is independent of both $P$ and the choice of $\Gamma$. We have also shown that

$$
f_{x}(Q)=f_{1}(Q)
$$

wherever the former is defined.

Recalling (3), that $m S_{1}=m S$, and the Riesz-Fischer Theorem, we obtain that

$$
\begin{aligned}
0 & =\lim _{n \rightarrow \infty} \iint_{S}\left|k_{x}^{(n)}-f_{1}\right|^{2} d S=\lim _{n \rightarrow \infty} \iint_{S_{1}}\left|k_{x}^{(n)}-f_{1}\right|^{2} d S \\
& =\lim _{n \rightarrow \infty} \iint_{S_{1}}\left|k_{x}^{(n)}-f_{x}\right|^{2} d S=\lim _{n \rightarrow \infty} \iint_{S}\left|k_{x}^{(n)}-f_{x}\right|^{2} d S,
\end{aligned}
$$

as well as the fact that $f_{x}$ has a summable square. These two facts readily yield, by Schwarz's Inequality, that

$$
\begin{aligned}
0 & \leqq \liminf _{n \rightarrow \infty}\left(\iint_{S}\left|f_{x}^{(n)}-f_{x}\right|^{2} d S\right)^{1 / 2} \\
& \leqq \limsup _{n \rightarrow \infty} \lim _{m \rightarrow \infty}\left(\iint_{S}\left|f_{x}^{(n)}-k_{x}^{(m)}+k_{x}^{(m)}-f_{x}\right|^{2} d S\right)^{1 / 2} \\
& \leqq \limsup _{n \rightarrow \infty} \limsup _{m \rightarrow \infty}\left(\iint_{S}\left|f_{x}^{(n)}-k_{x}^{(m)}\right|^{2} d S\right)^{1 / 2}+\left(\iint_{S}\left|k_{x}^{(m)}-f_{x}\right|^{2} d S\right)^{1 / 2} \\
& =\lim _{n, m \rightarrow \infty}\left(\iint_{S}\left|f_{x}^{(n)}-k_{x}^{(m)}\right|^{2} d S\right)^{1 / 2}+0=0 .
\end{aligned}
$$

In a similar fashion we may establish the existence of two sets $S_{2}, S_{0}$, $S_{2} \subseteq S, S_{0} \subseteq S, m S_{2}=m S_{0}=m S$, and of two functions $f_{v}$ and $f$, such that

$$
f_{y}(Q)=f_{2}\left(P_{2}\right)+\int_{\Gamma} f_{2,1} d x+f_{2,2} d y, \quad \lim _{n \rightarrow \infty} \iint_{S}\left|f_{y}^{(n)}-f_{y}\right|^{2} d S=0,
$$

and 


$$
f \equiv f\left(P_{3}\right)+\int_{\Gamma} f_{1} d x+f_{2} d y, \quad \lim _{n \rightarrow \infty} \iint_{S}\left|f^{(n)}-f\right|^{2} d S=0 .
$$

Now if a sequence of functions, defined on a finite linear interval, converges in the mean, and if a second sequence of functions, each of which is an indefinite integral of the corresponding function in the first set, is such that it converges at a single point, the second sequence converges in the mean.

This proves that $C_{x}^{\prime}$ can be taken in such a manner that $C_{x}^{\prime} \supseteq C_{x, x}$ when one considers the defining equations above and their analogue in the proof of the existence of $f$. Hence $C_{x} \subseteq C_{x, x}$. In a similar manner, we take $C_{y}^{\prime}$ in such a way that $C_{y}^{\prime} \supseteq C_{y, y}$ and obtain that $C_{y} \supseteq C_{y, y}$.

We also have, since for $y=c, c \in C_{x, x}, f_{1}=f_{x}$ is continuous, that

$$
\frac{\partial f}{\partial x}(x, c)=f_{1}=f_{x} \text {. }
$$

Similarly, for a point whose abscissa is in $C_{\nu, y}$,

$$
\frac{\partial f}{\partial y}=f_{2}=f_{y} .
$$

Finally we see that, on each line of $S_{1}$, almost everywhere

$$
\frac{\partial^{2} f}{\partial x^{2}}=f_{1,1}, \quad \frac{\partial^{2} f}{\partial x \partial y}=f_{1,2} .
$$

These results yield, upon inspection, that $f$ is of class $H$ on $S$ and that

$$
\lim _{n \rightarrow \infty}\left\|f^{(n)}-f\right\|=0
$$

irrespective of any further extension of the definition of $f$.

7. THE SPACE $\mathfrak{W}$

Let us define the inner product of two functions of class $H$ on $S$ as follows: $(u, v)=\iint_{S}\left(u \bar{v}+u_{x} \bar{v}_{x}+u_{\imath} \bar{v}_{y}+u_{x, x} \bar{v}_{x, x}+u_{x, y} \bar{v}_{x, y}+u_{y, x} \bar{v}_{y, x}+u_{y, y} \bar{y}_{y, y}\right) d S$.

We wish to set up a Hilbert space by means of functions of class $H$ on $S$ and the above inner product just as this is done in the classical theory, with $\mathfrak{R}_{2}$ and $\iint u \bar{v} d S$. As is the case of $\mathfrak{R}_{2}$, we are forced to take as our points classes of equivalent functions.

We set up the classes by means of the following statement: Two functions $u$ and $v$ belong to the same class if and only if 


$$
\|u-v\|=0 \text {. }
$$

We define the sum of two classes as the class to which the sum of an element of the first class and an element of the second class belongs. The product of a class and a number $c$ is the class to which $c$ times a function of the class belongs and the inner product of two classes $f$ and $g$ is defined as

$$
(f, g)=(u, v)
$$

where $u$ and $v$ belong to $f$ and $g$ respectively. One can readily show by familiar methods that these definitions yield a unique result in each case.

THEOREM VIII.* The space $\mathfrak{W}$, whose elements are the classes defined above with the above definition of addition, scalar multiplication, and inner product, is a Hilbert space. $†$

The completeness of $\mathfrak{B}$ is the lemma of the preceding section. The remainder of the proof is immediate and will be omitted.

Turning now to the consideration of equation (A) for functions $u$ of class $H$, we have, by Schwarz's Inequality and the boundedness of the coefficients,

$$
\begin{aligned}
\text { (a) } \iint|L(u)|^{2} d S \leqq \iint_{S}\left(\left|A_{20}\right|^{2}+2\left|A_{1,1}\right|^{2}+\cdots+\left|A_{0,0}\right|^{2}\right) \\
\cdot\left(\left|u_{x x}\right|^{2}+\left|u_{x, y}\right|^{2}+\cdots+|u|^{2}\right) d S \leqq C^{2}\|u\|^{2},
\end{aligned}
$$

where $C$ is a constant.

Thus we see that the functions $L(u), u \in H_{s}$, have a summable square on $S$. We can set up the usual space $\mathfrak{R}_{2}$ (cf. (S) Theorem 1.24) and corresponding to the set of $L(u)$ 's we have a subset of $\Omega_{2}$. Let $\Omega$ be the closed linear manifold determined by this subset. We suppose that $\Omega$ is of infinite dimensionality and thus is a Hilbert space (cf. (S) Theorem 1.18).

The equation (A) determines a transformation $T$ from $\mathfrak{B}$ to $\Omega$ defined as follows: If $f \in \mathfrak{B}$ and $u$ is one of its functional representatives, i.e., $u \in f$, then

- For other examples of spaces proposed for the study of partial differential equations, there is that of O. Nikodym (Journal de Mathématiques, vol. 9 (1933), pp. 95-109) who uses as his scalar product

$$
(f, g)=\iint_{S}\left(f_{x} \bar{g}_{x}+f_{y} \bar{g}_{y}+f_{z} \bar{g}_{z}\right) d S
$$

and obtains a simple proof of a theorem of M. Zaremba.

Spaces of the Banach type have been effectively used by J. Schauder (Mathematische Annalen, vol. 106 (1932), pp. 661-721) in the study of elliptic partial differential equations. Hyperbolic differential equations of a special form with initial conditions have been treated by $D$. C. Lewis (these Transactions, vol. 35 , pp. 792-823) by methods which involve $\mathfrak{l}_{p}$.

The work of Ritz can be regarded as having to do with spaces in which the length of a function $u$ is the integral $J$ which he minimizes.

$\dagger(\mathrm{S})$ Definition 1.1. 
from the above we see that $L(u)$ is a member of a class $g$, which is a point in $\Omega$, and we define $T f=g$. Thus it follows from $(\alpha)$ that $T$ is a limited transformation with domain $\mathfrak{B}$ and hence $T$ is closed. The range of $T$ is everywhere dense in $\Omega$, by virtue of the definition of $\Omega$.

\section{APPlication to DiffERENTIAL OPERATORS}

We are now in a position to apply our results on transformations between Hilbert spaces to the transformation represented by equation (A). Specifically we give the significance in terms of functions of Theorems 1.16 and II, which we may apply since we have restricted our range space to the closed linear manifold $\Omega$, in $\mathfrak{R}_{2}$, determined by the range of $T$.

The following notation is useful.

Definition III. $w={ }_{m} u$ is to mean that $w=u$ almost everywhere on $S$. If $f_{i} \in H_{s}$ and if the sequence $\left\{f_{i}\right\}$ is such that

$$
\lim _{m+n \rightarrow \infty}\left\|\sum_{1}^{n} f_{i}-\sum_{1}^{m} f_{i}\right\|=0
$$

then $\sum_{1}^{\infty} f_{i}$ is to denote the function whose existence is proved by the lemma of $\$ 6$.

$u^{\prime}=_{m+} u^{\prime \prime}$ is to mean $\left\|u^{\prime}-u^{\prime \prime}\right\|=0$.

We then obtain

THEOREM 1.16'. Given the equation (A) of the introduction, and a bounded connected region $S$. There exist two orthonormal sets of functions $\left\{u_{i}\right\}$ and $\left\{v_{i}\right\}, u_{i}$ and $v_{i} \in H_{a}$, such that the corresponding elements in $\mathfrak{B}$ are mutually orthogonal and are such that

(1) a necessary and sufficient condition that $u \in H_{s}$ be such that $L(u)={ }_{m} 0$, is that

$$
u={ }_{m+} \sum_{1}^{\infty}\left(u, u_{i}\right) u_{i}, \quad \sum_{1}^{\infty}\left|\left(u, u_{i}\right)\right|^{2}<\infty ;
$$

(2) if $v={ }_{m+} \sum_{1}^{\infty}\left(v, v_{i}\right) v_{i}$ and $L(v)={ }_{m} 0$, then $v={ }_{m+} 0$;

(3) if $w \in H_{s}$, then

$$
w={ }_{m+} \sum_{1}^{\infty}\left(w, u_{i}\right) u_{i}+\sum_{1}^{\infty}\left(w, v_{i}\right) v_{i}, \sum_{1}^{\infty}\left|\left(w, u_{i}\right)\right|^{2}+\sum_{1}^{\infty}\left|\left(w, v_{i}\right)\right|^{2}<\infty .
$$

This is of course Theorem 1.16 applied to this special case and then using the fact that in any closed linear manifold there exists an orthonormal set complete in the closed linear manifold, for $\mathfrak{N}$ and $\mathfrak{S} \ominus \mathfrak{N}((\mathrm{S})$ Theorems 1.19 and 1.14). If we have given a complete orthonormal set $\left\{f_{i}\right\}$ in $\mathfrak{B}$ and a 
complete orthonormal set $\left\{g_{i}\right\}$ in $\Omega$ we can actually exhibit the sets $u_{i}$ and $\nu_{i}$. For

$$
T^{*} g_{i}=\sum_{j=1}^{\infty}\left(T^{*} g_{i}, f_{j}\right)_{w} f_{i}=\sum_{1}^{\infty}\left(g_{i}, T f_{j}\right)_{k} f_{j}
$$

and $\left\{T^{*} g_{i}\right\}$ determine $\mathfrak{E} \ominus \mathfrak{N}$ by Theorems II and 1.16 , and by the method in the note on construction, we can obtain a set which determines $\Re$.

We define $L^{*}(v)$ for any $v$ of a class $g$, which is an element of $\Re$, as a function of class $H$ which is a member of $T^{*} g$.

THEOREM II'. Let $\left\{\psi_{i}\right\}$ denote the set obtained by orthonormalizing the set $\left\{L^{*}\left(L\left(v_{i}\right)\right)\right\}$ where the $v_{i}$ are as in Theorem 1.16'. Then $\left\{\psi_{i}\right\}$ determines the the same closed linear manifold as the set $\left\{v_{i}\right\}$ of Theorem $1.16^{\prime}$. For each $\psi_{i}$ there is a $\chi_{i}$ such that $L^{*}\left(\chi_{i}\right)={ }_{m+} \psi_{i}$. A necessary and sufficient condition that a function $w$, a member of an element $g$ of $\Omega$, be such that there is a $u \in H_{s}$ for which $L(u)={ }_{m} w$, is that

$$
\sum_{1}^{\infty}\left|\left(w, x_{i}\right)\right|^{2}<\infty
$$

when w satisfies this condition and

then $L(v)={ }_{m} w$.

$$
v={ }_{m+} \sum_{1}^{\infty}\left(w, \chi_{i}\right) \psi_{i}
$$

This is just the application of Theorem II to the special case treated here.

Theorem II' offers a generalization of the methods of Ritz $\dagger$ in the sense that it generalizes the formulas upon which actual calculations would be based.

We have restricted the coefficients to be bounded and upon this assumption the limitedness of this transformation was shown. In the unbounded cases, some knowledge of the resolution of the identity corresponding to $T^{*} T$ must be had before results analogous to those of Theorems $1.16^{\prime}$ and $\mathrm{II}^{\prime}$ can be obtained.

The following examples indicate what results are to be expected in applying the above methods. Let us suppose $S$ is a square of side $2 \pi$ with sides parallel to an axis. We will show that the range of the transformation associated with

$$
L(u)=\frac{\partial^{2} u}{\partial x^{2}}+\frac{\partial^{2} u}{\partial y^{2}}=v
$$

is the whole space $\mathfrak{R}_{2}$. Let $v$ be a function of summable square; then

$\dagger$ Loc. cit. 


$$
v=\sum_{n=-\infty}^{\infty} \sum_{m=-\infty}^{\infty} a_{n, m} \frac{e^{i n x+i m y}}{2 \pi} ; a_{n, m}=\frac{1}{2 \pi} \iint_{S} e^{-i n x-i m y} v d S .
$$

Now when we orthonormalize the sequence $\left\{e^{i n x+i m y}\right\}$ in $\mathfrak{B}$ we obtain

$$
\left\{\frac{1}{2 \pi}\left(1+m^{2}+n^{2}+\left(m^{2}+n^{2}\right)^{2}\right)^{-1 / 2} e^{i n x+i m y}\right\} \text {. }
$$

Now since $\sum_{-\infty}^{\infty} \sum_{-\infty}^{\infty}\left|a_{n, m}\right|^{2}<\infty$,

$$
\sum_{n^{2}+m^{2}>0}\left|a_{n, m}\right|^{2} \frac{1+n^{2}+m^{2}+\left(m^{2}+n^{2}\right)^{2}}{\left(m^{2}+n^{2}\right)^{2}}<\infty,
$$

let

$$
\begin{aligned}
u & =\sum_{n^{2}+m^{>}>0} a_{n, m} \frac{\left(1+n^{2}+m^{2}+\left(m^{2}+n^{2}\right)^{2}\right)^{1 / 2}}{m^{2}+n^{2}} \frac{1}{2 \pi} \frac{e^{i n x+i m y}}{\left(1+n^{2}+m^{2}+\left(n^{2}+m^{2}\right)^{2}\right)^{1 / 2}} \\
& =\sum_{n^{2}+m^{2}>0} \frac{a_{n, m}}{2 \pi} \frac{e^{i n x+i m y}}{m^{2}+n^{2}} .
\end{aligned}
$$

From (1), we see that $u \in H_{\text {s }}$ and $a_{00} x^{2} /(4 \pi)+u \epsilon H_{\text {s }}$ and hence

$$
L_{1}\left(\frac{a_{00} x^{2}}{4 \pi^{2}}+u\right)={ }_{m} v .
$$

Thus when $T_{1}$ is restricted to $\mathfrak{B} \ominus \mathfrak{N}$ so that it has an inverse, Theorem 1.25 yields that this inverse is bounded.

However, for the same square in the case of

$$
L_{2}(u)=\frac{\partial^{2} u}{\partial x \partial y}=v
$$

while the range of $T$ is everywhere dense in $\mathfrak{R}_{2}$, it is not $\mathfrak{R}_{2}$. For we have no difficulty in finding a $u_{n, m}$ such that

$$
\frac{\partial^{2} u_{n, m}}{\partial x \partial y}=e^{i n x+i m y},
$$

thus showing that the range determines $\mathbb{R}_{2}$ and since it is a linear manifold that it is everywhere dense in $\mathfrak{R}_{2}$.

However there is no $u \in \mathfrak{W}$ such that

$$
\frac{\partial^{2} u}{\partial x \partial y}=\log x
$$

although $\log x \in \mathfrak{R}_{2}$. For if $u$ satisfies (2) it is in the form 


$$
u=x y(\log x-1)+X(x)+Y(y),
$$

and one can easily show that $\partial^{2} u / \partial x^{2}$ does not have a summable square.

9. Boundary Values of $f_{x}$ AND $f_{y}$

In the remaining sections, we give a method for the solution of boundary value problems, specifically the problem formulated in $\$ 10$. We must first restrict $S$ so as to have a boundary which fulfils the following conditions.

Let the boundary $B$ of $S$ be a rectifiable curve such that a polygon $P_{1} P_{2} \cdots P_{n}$, $P_{i}=\left(x_{i}, y_{i}\right)$, with $P_{i} P_{i+1}$ parallel to an axis and the side of a rectangle whose interior is in $S$ and with the interior of $P_{1} P_{2} \cdots P_{n}$ in $S$, can be constructed with certain vertices on $B$ in such a manner that the arc of $B$ between two vertices which are successive along the curve is such that either

(1) it is intercepted by two alternate vertices of the polygon $P_{i}$ and $P_{i+2}$ and if $(x, y)$ is a point of the arc $P_{i} P_{i+2}$, there is a relationship $y=Y^{\prime}(x)$, which is such that $Y^{\prime}$ is monotonic and single-valued except possibly at a denumerable set of points, where the continuum of values between the limits on the right and left are assumed, or

(2) it is intercepted by two successive vertices $P_{j}$ and $P_{j+1}$ and (a) $x_{j}=x_{j+1}$ and the $\operatorname{arc} P_{j} P_{j+1}$ is given by an equation $x=X(y), y_{j} \leqq y \leqq y_{j+1}$, where $X(y)$ is single-valued and has bounded Dini derivatives or (b) $y_{j+1}=y_{j}$ and the arc $P_{j} P_{i+1}$ is given by the equation $y=Y(x)$ where $Y$ is single-valued and has bounded Dini derivatives. (See the figure.)

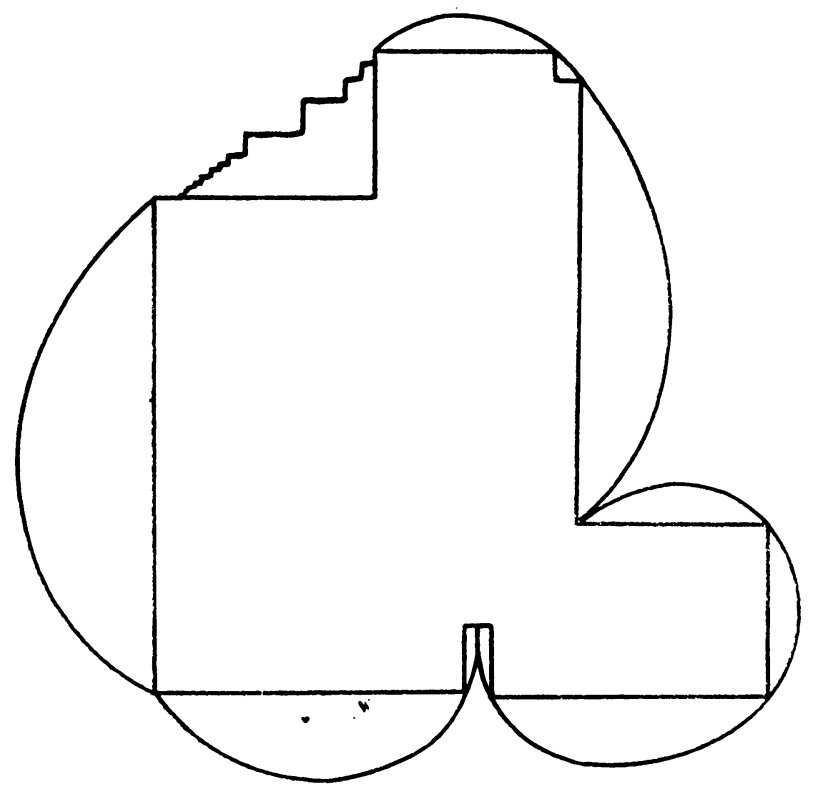


As to the relationship between conditions (1) and (2) it may be pointed out that a monotonic curve may be rotated into one with bounded Dini derivatives. It should also be pointed out that these restrictions rule out comparatively smooth curves with outward pointing cusps.*

We shall suppose from now on that $S$ satisfies the above conditions and that $f(x, y) \in H_{s}$.

Lemma I. Let $P_{i} P_{i+1}$ be a segment of the polygon described above. Let us suppose that $y_{i}=y_{i+1}$ and $x_{i}<x_{i+1}$; then $\lim _{y \rightarrow y_{i}} f_{x}(x, y)$ exists for almost every $x$ such that $x_{i} \leqq x \leqq x_{i+1}$. If $x \in \Gamma_{x}$ and $\left(x, y_{i}\right) \epsilon S$, then $\lim _{y \rightarrow y_{i}} f_{x}(x, y)=f_{x}\left(x, y_{i}\right)$. $\lim _{y \rightarrow y_{i}} f_{x}(x, y)$ is measurable and has summable square on the interval $x_{i} \leqq x \leqq x_{i+1}$. There exists a constant $K_{i}$ such that

$$
\int_{x_{i}}^{x_{i+1}}\left|\lim _{y \rightarrow y_{i}} f_{x}(x, y)\right|^{2} d x \leqq K_{i}\|f\|^{2} .
$$

Now by the specifications on the polygon $P_{1} P_{2} \cdots P_{n}$ it is possible to find a rectangle $\Delta=P_{i} P_{i+1} A B, A=\left(x_{i+1}, a\right), B=\left(x_{i}, a\right)$, whose interior is in $S$. Let us suppose $a<y_{i}$. Now

$$
\iint_{\Delta}\left|f_{x}\right|^{2} d S
$$

exists. By Fubini's Theorem,

$$
\int_{a}^{y_{i}}\left(\int_{x_{i}}^{x_{i+1}}\left|f_{x}(x, y)\right|^{2} d x\right) d y
$$

exists. Therefore there is a $y^{\prime}, a \leqq y^{\prime}<y$, such that

$$
\int_{x_{i}}^{x_{i+1}}\left|f\left(x, y^{\prime}\right)\right|^{2} d x \leqq \frac{1}{y_{i}-a} \int_{a}^{y_{i}} \int_{x_{i}}^{x_{i+1}}\left|f_{x}(x, y)\right|^{2} d x d y \leqq K^{(1)}\|f\|^{2} .
$$

Now let $\Delta^{\prime}$ be the rectangle with vertices $P_{i}, P_{i+1},\left(x_{i+1}, y^{\prime}\right),\left(x_{i}, y^{\prime}\right)$. Now $\Delta^{\prime} \subseteq \Delta$ and we have by Fubini's Theorem

$$
\iint_{\Delta^{\prime}}\left|f_{x, y}\right|^{2} d S=\int_{x_{i}}^{x_{i+1}} \int_{y^{\prime}}^{y_{i}}\left|f_{x, y}\right|^{2} d y d x,
$$

and since the summability of the square on a bounded set implies summability, we have

* However, for our purposes, some outward pointing cusps must be excluded as the following example shows. Let $S$ be the region of points $(x, y)$ such that $0<x<1, x^{8}>y>0$. One can easily verify that $u^{1 / 2} \in H_{a}$, and also that the values of $u_{x}$ on the boundary do not have a summable square with respect to integration along the arc-length. 


$$
\iint_{\Delta^{\prime}} f_{x, y} d S
$$

exists. On applying Fubini's Theorem, we have that for almost every $x$, $x_{i} \leqq x \leqq x_{i+1}$, the integral

$$
\int_{y^{\prime}}^{y_{i}} f_{x, y}(x, y) d y
$$

exists and is a measurable function of $x$ for $x_{i} \leqq x \leqq x_{i+1}$. Now

$$
\left|\int_{y^{\prime}}^{y_{i}} f_{x}(x, y) d y\right|^{2} \leqq\left(y_{i}-y^{\prime}\right) \int_{y^{\prime}}^{y_{i}}\left|f_{x, y}(x, y)\right|^{2} d y .
$$

The existence of the integral on the right of (4) now implies that the function of $x$ on the left of (7) is summable and hence the function (6) has a summable square and

$$
\begin{aligned}
& \int_{x_{i}}^{x_{i+1}}\left|\int_{y^{\prime}}^{y_{i}} f_{x, y}(x, y) d y\right|^{2} d x \\
& \quad \leqq\left|y_{i}-y^{\prime}\right| \int_{x_{i}}^{x_{i+1}} \int_{y^{\prime}}^{y_{i}}\left|f_{x, y}(x, y)\right|^{2} d y d x \\
& \quad=\left|y_{i}-y^{\prime}\right| \iint_{\Delta^{\prime}}\left|f_{x, y}\right|^{2} d S \leqq K^{\prime}\|f\|^{2} .
\end{aligned}
$$

Now for $x \in \Gamma_{x}$ and for which (6) is defined and hence for almost every $x$ such that $x_{i} \leqq x \leqq x_{i+1}$, we have

$$
\begin{aligned}
\lim _{y \rightarrow y_{i}} f_{x}(x, y) & =\lim _{y \rightarrow y_{i}}\left(f_{x}\left(x, y^{\prime}\right)+\int_{y^{\prime}}^{y} f_{x, y}(x, \xi) d \xi\right) \\
& =f_{x}\left(x, y^{\prime}\right)+\int_{y^{\prime}}^{y_{i}} f_{x, y}(x, \xi) d \xi .
\end{aligned}
$$

Hence $\lim _{y \rightarrow y_{i}} f_{x}(x, y)$ exists for almost every $x$ such that $x_{i} \leqq x \leqq x_{i+1}$ and is a measurable function with a summable square on the interval $x_{i} \leqq x \leqq x_{i+1}$, since it is the sum of two functions which are measurable and have summable squares. Then from (4) and (8), by using the triangle theorem, we obtain

$$
\int_{x_{i}}^{x_{i+1}}\left|\lim _{y \rightarrow y_{i}} f_{x}(x, y)\right|^{2} d x \leqq K_{i}^{\prime}\|f\|^{2}
$$


Lemma II. Let $\operatorname{arc} P_{i} P_{i+2}$ be an arc of the curve $B$ such that case (1) of the specifications of $B$ applies. Let us suppose that $x_{i}<x_{i+1}=x_{i+2}$ and $y_{i}=y_{i+1}$ $<y_{i+2}$. Let $Y(x)$ be defined as the least of the numbers $Y^{\prime}(x)$ for $x$ fixed. ${ }^{*}$ Then obviously $Y(x)$ is a monotonically increasing single-valued function of $x$, and if $f_{x}(x, Y(x))$ is defined as the $\lim _{y \rightarrow Y(x)} f_{x}(x, y)$, when the latter exists, then $f_{x}(x, Y(x))$ is defined almost everywhere on the interval $x_{i} \leqq x \leqq x_{i+2}$ and is a measurable function of $x$ with a summable square on this interval, and there is a $K$ such that

$$
\int_{x_{i}}^{x_{i+2}}\left|f_{x}(x, Y(x))\right|^{2} d x \leqq K\|f\|^{2} .
$$

Let us denote the rectangle having $P_{i} P_{i+1}$ and $P_{i+1} P_{i+2}$ among its sides as $\Delta$. Then

$$
\iint_{\Delta \cdot S}\left|f_{x, y}\right|^{2} d S
$$

exists. Hence by Fubini's Theorem

$$
\int_{y_{i}}^{Y(x)}\left|f_{x, y}(x, y)\right|^{2} d y
$$

exists for almost every $x$ such that $x_{i} \leqq x \leqq x_{i+2}$ and is a summable function of $x$ such that

$$
\int_{x_{i}}^{x_{i+1}} d x \int_{y_{i}}^{Y(x)}\left|f_{x, y}(x, y)\right|^{2} d y=\iint_{\Delta \cdot S}\left|f_{x, y}\right|^{2} d y .
$$

Now since the summability of the square implies summability, the existence of (1) implies the existence of

$$
\iint_{\Delta \cdot s} f_{x, y} d S
$$

and by Fubini's Theorem

$$
\int_{y_{i}}^{Y(x)} f_{x, y}(x, y) d x
$$

exists for almost every $x$ such that $x_{i} \leqq x \leqq x_{i+2}$ and is a measurable function on this interval.

Furthermore by Schwarz's Inequality when (2) and (5) exist, which is for almost every $x$ in the interval $x_{i} \leqq x \leqq x_{i+2}$,

* It is to be remembered that $Y^{\prime}(x)$ is not necessarily single-valued. 


$$
\begin{aligned}
\left|\int_{y_{i}}^{Y(x)} f_{x, y}(x, y) d y\right|^{2} & \leqq\left|Y(x)-y_{i}\right| \int_{y_{i}}^{Y(x)}\left|f_{x, y}(x, y)\right|^{2} d y \\
& \leqq\left|y_{i+2}-y_{i}\right| \int_{y_{i}}^{Y(x)}\left|f_{x, y}(x, y)\right|^{2} d y .
\end{aligned}
$$

Since the function on the right is summable, the function on the left is also, and furthermore integrating and using (3) we obtain

$$
\begin{aligned}
\int_{x_{i}}^{x_{i+2}}\left|\int_{y_{i}}^{Y(x)} f_{x, y}(x, y) d y\right|^{2} & \leqq\left|y_{i+2}-y_{i}\right| \iint_{\Delta \cdot s}\left|f_{x, y}\right|^{2} d S \\
& \leqq\left|y_{i+2}-y_{i}\right|\|f\|^{2} .
\end{aligned}
$$

Remembering that for $Y(x)=y_{i}$, by Lemma $\mathrm{I}, f_{x}(x, Y(x))$ exists for almost every such $x$, we obtain for all $x$ such that $x \in \Gamma_{x}$ and for which (5) has been shown to hold and hence for almost every $x$ such that $x_{i} \leqq x \leqq x_{i+2}$,

$$
\begin{aligned}
f_{x}(x, Y(x)) & =\lim _{y \rightarrow Y(x)^{-}} f_{x}(x, y) \\
& =\lim _{y \rightarrow Y(x)^{-}}\left(f_{x}\left(x, y_{i}\right)+\int_{y i}^{y} f_{x, y}(x, \eta) d \eta\right) \\
& =f_{x}\left(x, y_{i}\right)+\int_{y_{i}}^{Y(x)} f_{x, y}(x, \eta) d \eta .
\end{aligned}
$$

Thus for such an $x, f_{x}(x, Y(x))$ has been defined. It is known from Lemma I that $f_{x}\left(x, y_{i}\right)$ is measurable and has a summable square and there is a $K_{i}$ such that

$$
\int_{x_{i}}^{x_{i+1}}\left|f\left(x, y_{i}\right)\right|^{2} d x \leqq K_{i}\|f\|^{2} .
$$

Now from (7) $f_{x}(x, Y(x))$ is a measurable function of $x$ with a summable square, since it is the sum of two functions having this property, and from (6), (7) and (8) using the triangle theorem, we obtain

$$
\int_{x_{i}}^{x_{i+1}}\left|f_{x}(x, Y(x))\right|^{2} d x \leqq K\|f\|^{2} .
$$

Since $Y^{\prime}(x)$ is monotonically increasing, it follows that if $(x, y) \epsilon \operatorname{arc} P_{i} P_{i+2}$, then there is a relationship $x=X^{\prime}(y)$ between the coordinates, which is also monotonically increasing and single-valued except possibly for a denumerable set of points, where all values between the limits on the right and left are assumed. 
Lemma III. Let arc $P_{i} P_{i+2}$ be as in Lemma II. Let $X(y)$ be defined as the greatest number $x$, such that $Y(x)=y . X(y)$ is a single-valued and monotonically increasing function of $y$. Let $f_{x}(X(y), y)$ be defined as $\lim _{x \rightarrow X(y)}{ }^{+} f_{x}(x, y)$ when the latter exists. Then $f_{x}(X(y), y)$ is defined almost everywhere for the interval $y_{i} \leqq y \leqq y_{i+2}$ and on this interval is measurable and has a summable square and there is a $K$ such that

$$
\int_{y_{i}}^{y_{i+2}}\left|f_{x}(X(y), y)\right|^{2} d y \leqq K\|f\|^{2}
$$

The proof is, by the remark which precedes the lemma, quite analogous to the proof of Lemma II.

Lemma IV. If $\left(x_{0}, y_{0}\right) \epsilon \operatorname{arc} P_{i} P_{i+2}$ is such that $f_{x}\left(x_{0}, Y\left(x_{0}\right)\right)$ and $f_{x}\left(X\left(y_{0}\right)\right.$, $y_{0}$ ) both exist, then $f_{x}\left(x_{0}, Y\left(x_{0}\right)\right)=f_{x}\left(X\left(y_{0}\right), y_{0}\right)$.

First suppose that $y_{0} \neq y_{i}$. Now since

$$
f_{x}\left(x_{0}, Y\left(x_{0}\right)\right)=\lim _{y \rightarrow\left(x_{0}\right)^{-}} f_{x}\left(x_{0}, y\right),
$$

given an $\epsilon>0$, we can find a $\delta^{\prime}$ such that for $Y\left(x_{0}\right)-y \geqq Y\left(x_{0}\right)-\delta^{\prime}$

$$
\left|f_{x}\left(x_{0}, Y\left(x_{0}\right)\right)-f_{x}\left(x_{0}, y\right)\right| \leqq \epsilon .
$$

Similarly we can find a $\delta^{\prime \prime}$ such that for $X\left(y_{0}\right)<x \leqq X\left(y_{0}\right)+\delta^{\prime \prime}$,

$$
\left|f_{x}^{\prime}\left(X\left(y_{0}\right), y_{0}\right)-f_{x}\left(x, y_{0}\right)\right| \leqq \epsilon .
$$

Now for almost every $x$ such that $x_{i} \leqq x \leqq x_{i+2}$ and all $y$ such that $y_{0}>y \geqq y_{i}$,

$$
\begin{aligned}
\left|f_{x}(x, y)-f_{x}\left(x, y_{0}\right)\right|^{2} & =\left|\int_{y_{0}}^{y} f_{x, y}(x, \xi) d \xi\right|^{2} \\
& \leqq\left.\left|y_{0}-y\right|\left|\int_{y_{0}}^{y_{y}}\right| f_{x, y}(x, \xi)\right|^{2} d \xi \mid .
\end{aligned}
$$

Let

$$
g(x, y)=\left.\left|\int_{y_{0}}^{y}\right| f_{x, y}(x, \xi)\right|^{2} d \xi \mid .
$$

Now $g(x, y) \geqq 0$. Let $\Delta_{y}$ be the rectangle $x_{0} \leqq x \leqq x_{i+2}, y \leqq \xi<y_{0}$. Let

$$
\begin{aligned}
F(y) & =\int_{x_{0}}^{x_{i+2}} g(x, y) d x=\left.\int_{x_{0}}^{x_{i+2}}\left|\int_{y_{0}}^{y}\right| f_{x, y}(x, \xi)\right|^{2} d \xi \mid d x \\
& =\iint_{\Delta_{y}}\left|f_{x, y}\right|^{2} d S=\left.\left|\int_{y_{0}}^{y} d \xi \int_{x_{0}}^{x_{i+2}}\right| f_{x, y}(x, \xi)\right|^{2} d x \mid .
\end{aligned}
$$


Then (5) shows that $F(y) \rightarrow 0$ as $y \rightarrow y_{0}$. We also have, for $y$ fixed,

$$
m_{x}\left\{\left|y-y_{0}\right| g(x, y) \geqq F(y)^{1 / 2}\right\} \leqq\left|y_{0}-y\right| F(y)^{1 / 2} \text {. }
$$

Now since $F(y) \rightarrow 0$ as $y \rightarrow y_{0}$, we can choose a $\delta^{\prime \prime \prime}>0$ in such a way that $F(y)^{1 / 2}<\epsilon$ for $\left|y-y_{0}\right|<\delta^{\prime \prime \prime}$.

Now similarly for almost every $y$ such that $y_{0}>y \geqq y_{i}$ and all $x$ such that $x_{0}<x \leqq x_{i+2}$, we have by the Schwarz inequality,

Let

$$
\begin{aligned}
\left|f_{x, y}(x, y)-f_{x}\left(x_{0}, y\right)\right|^{2} & =\left|\int_{x_{0}}^{x} f_{x, x}(\eta, y) d \eta\right|^{2} \\
& \leqq\left|x-x_{0}\right| \int_{x_{0}}^{x}\left|f_{x_{0} x}(\eta, y)\right|^{2} d \eta .
\end{aligned}
$$

$$
\begin{gathered}
h=\int_{x_{0}}^{x}\left|f_{x, x}(\eta, y)\right|^{2} d \eta, \\
G(x)=\int_{y_{i}}^{y_{0}} d y h(x, y)=\int_{y_{i}}^{y_{0}} d y \int_{x_{0}}^{x}\left|f_{x, x}(\eta, y)\right|^{2} d \eta \\
=\iint_{\Delta_{x}}\left|f_{x, x}(\eta, y)\right|^{2} d S,
\end{gathered}
$$

where $\Delta_{x}$ is the rectangle $\left\{x_{0} \leqq \eta \leqq x, y_{i} \leqq y \leqq y_{0}\right\}$. Now as above $G(x) \rightarrow 0$ as $x \rightarrow x_{0}$, and we can choose a $\delta^{\text {iv }}$ such that for $x_{0}<x \leqq x_{0}+\delta^{\text {iv }}, G(x)^{1 / 2} \leqq \epsilon$. Let us suppose that $\epsilon<\frac{1}{4}$. Let $\delta=\min \left(\delta^{\prime}, \delta^{\prime \prime}, \delta^{\prime \prime \prime}, \delta^{\text {iv }}\right)$ and $\Delta$ be the rectangle $\left\{x_{0}<x \leqq x_{0}+\delta, y_{0}-\delta \leqq y<y_{0}\right\}$. Then from (6) by Fubini's Theorem

$$
\begin{gathered}
m_{x, y}\left\{\Delta\left\{\left|y-y_{0}\right| g(x, y) \geqq \epsilon\right\}\right\} \leqq \int_{y_{0}-8}^{y_{x}} m_{x}\left\{\left|y-y_{0}\right| g(x, y) \geqq \epsilon\right\} d y \\
\leqq \int_{y_{0}-8}^{y_{0}}\left|y-y_{0}\right| F(y)^{1 / 2} d y \leqq \int_{y_{0}-\delta}^{y_{0}} \delta \epsilon d y \leqq \delta^{2} \epsilon<\frac{1}{2} \delta^{2} .
\end{gathered}
$$

Similarly

$$
m_{x, y}\left\{\Delta \cdot\left\{\left|x-x_{0}\right| h(x, y) \geqq \epsilon\right\}\right\}<\frac{1}{2} \delta^{2} .
$$

Hence the intersection $I^{\prime}$ of the three sets $\Delta$, the set for which

$$
\left|x-x_{0}\right| h(x, y)<\epsilon
$$

holds, and the set for which

$$
\left|y-y_{0}\right| g(x, y)<\epsilon
$$

holds, is by (9) and (10) such that $m I^{\prime} \geqq \frac{1}{3} \delta^{2}$. 
The intersection $I$ of $I^{\prime}$ and the sets for which (3) and (7) hold is not empty. Let $(x, y)$ be a point of $I$. From (3) and (12) we obtain

$$
\left|f_{x}(x, y)-f_{x}\left(x, y_{0}\right)\right| \leqq \epsilon^{1 / 2}
$$

from (7) and (11),

$$
\left|f_{x}(x, y)-f_{x}\left(x_{0}, y_{0}\right)\right| \leqq \epsilon^{1 / 2} .
$$

Since $\delta \leqq \min \left(\delta^{\prime}, \delta^{\prime \prime}\right)$, we can apply (1) and (2) which with (13) and (14) yield

$$
\left|f_{x}\left(x_{0}, Y\left(x_{0}\right)\right)-f_{x}\left(X\left(y_{0}\right), y_{0}\right)\right| \leqq 2 \epsilon+2 \epsilon^{1 / 2} .
$$

Since $\epsilon$ may be taken arbitrarily small we must have

$$
f_{x}\left(x_{0}, Y\left(x_{0}\right)\right)=f_{x}\left(X\left(y_{0}\right), y_{0}\right) .
$$

When $y_{i}=y_{0}$, we take for the interval for $y$, when (3) is to be considered, the interval $y_{0}>y \geqq y_{0}-\eta$, where $\eta$ is chosen so small that the set $\left(x_{0}, y\right)$ ( $y$ in the interval) is in the rectangle associated with $P_{i} P_{i+1}$. The proof is then quite similar to the above. When $x_{0}=x$ a similar consideration holds.

Definition IV. $f_{x}(P), P \in$ arc $P_{i} P_{i+2}$, is defined as $f_{x}(x, Y(x))$ when $P=(x, Y(x))$ and $f_{x}(P)=f_{x}(X(y), y)$ when $P=(X(y), y)$.

From the above lemma we see that this definition is consistent.

LEMMA V. $f_{x}(P)$ regarded as a function of the arc-length is defined for almost every $s$ which corresponds to a point of arc $P_{i} P_{i+2}$, and is measurable for this $s$ interval, and there is a $K$ such that

$$
\int_{\operatorname{arc} P_{i} P_{i+2}}\left|f_{x}(P)\right|^{2} d s \leqq K\|f\|^{2} .
$$

It is well known that for an arc such as $P_{i} P_{i+2}, x=x(s)$ and $y=y(s)$ are monotonic absolutely continuous functions of $s^{*}$ and that there exist two functions, $D_{s} x$ and $D_{s} y$, defined for every $s$ and such that

$$
x=x_{i}+\int_{s i}^{s} D_{s} x d s, \quad y=y_{i}+\int_{s i}^{s} D_{s} y d s
$$

also such that $D_{s} x \geqq 0$ and $D_{s} y \geqq 0, D_{s} x$ being one of the Dini derivatives, and it will be convenient to suppose in the case treated here that it is $D_{s}^{+} x$.

Now

$$
s_{1}-s_{0}=\lim \sup \sum_{1}^{N}\left(\Delta x_{i}^{2}+\Delta y_{i}^{2}\right)^{1 / 2},
$$

\footnotetext{
* Cf. Hobson, Functions of a Real Variable, pp. 338-341, especially p. 340, also pp. 596, 411.
} 
and since $x(s)$ and $y(s)$ are monotonic, $s_{1}-s_{0} \leqq \lim \sup \sum_{1}^{N}\left(\Delta x_{i}+\Delta y_{i}\right)=\lim \sup \left(x_{1}-x_{0}+y_{1}-y_{0}\right)=x_{1}-x_{0}+y_{1}-y_{0}$ or

$$
1 \leqq \frac{\Delta x}{\Delta s}+\frac{\Delta y}{\Delta s}
$$

Hence we have for every $s$

$$
1 \leqq D_{s} x+D_{s} y .
$$

Now*

$$
\begin{aligned}
& \int_{x_{i}}^{x_{i+2}}\left|f_{x}(P)\right|^{2} d x=\int_{\operatorname{arc} P_{i} P_{i+2}}\left|f_{x}(P)\right|^{2} D_{s} x d s, \\
& \int_{y_{i}}^{y_{i+2}}\left|f_{x}(P)\right|^{2} d y=\int_{\operatorname{arc} P_{i} P_{i+2}}\left|f_{x}(P)\right|^{2} D_{s} y d s,
\end{aligned}
$$

and $\left|f_{x}(P)\right|{ }^{2} D_{s} x$ and $\left|f_{x}(P)\right|{ }^{2} D_{s} y$ are measurable summable functions of $s$ defined for almost every $s$, corresponding to a point of arc $P_{i} P_{i+2}$. (They are defined as zero, when $D_{s} x$ and $D_{s} y$ are zero respectively.) Since $D_{s} x+D_{s} y$ is a measurable function of $s$ on the $s$ interval corresponding to $\operatorname{arc} P_{i} P_{i+2}$, $1 /\left(D_{s} x+D_{s} y\right)$ is bounded and measurable on this interval and hence

$$
\frac{1}{D_{s} x+D_{s} y}\left(\left|f_{x}(P)\right|^{2} D_{s} x+\left|f_{x}(P)\right|^{2} D_{s} y\right)=\left|f_{x}(P)\right|^{2}
$$

is defined almost everywhere and is measurable and summable. Furthermore by (2) and (3) and Lemmas II and III,

$$
\begin{aligned}
\int_{\operatorname{arc} P_{i} P_{i+2}}\left|f_{x}(P)\right|^{2} d s & \leqq \int_{\operatorname{arc} P_{i} P_{i+2}}\left|f_{x}(P)\right|^{2}\left(D_{s} x+D_{s} y\right) d s \\
& \leqq\left(K_{1}+K_{2}\right)\|f\|^{2} .
\end{aligned}
$$

If the argument of the preceding paragraph is repeated with $f_{x}(P)$ substituted for $\left|f_{x}(P)\right|^{2}$, since we have shown that $f_{x}(x, Y(x))$ and $f_{x}(X(y), y)$ are measurable functions of $x$ and $y$ respectively, in the appropriate interval, we shall obtain that $f_{x}(P)$ is a measurable function of $s$. This completes the proof of the lemma.

Let us suppose now that $\operatorname{arc} P_{j} P_{j+1}$ is an $\operatorname{arc}$ of $B$ for which case (2) holds. The proof of the following lemma is quite similar to the proofs of Lemmas II

* Cf. Hobson, loc. cit., p. 665. 
and III above and the reader will find no difficulty in making the slight adjustments necessary. We state it for case (2a).

Lemma VI. Let $\operatorname{arc} P_{j} P_{j+1}$ be such that (2a) holds. Then if $\lim _{x \rightarrow X(y)} f_{x}(x, y)$ for $y_{j} \leqq y \leqq y_{j+1},(x, y) \epsilon S$, is denoted by $f_{x}(X(y), y)$ when it exists, $f_{x}(X(y), y)$ is a measurable function of $y$ and there is $a K$ such that

$$
\int_{y_{j}}^{y_{j+1}}\left|f_{x}(X(y), y)\right|^{2} d y \leqq K\|f\|^{2} .
$$

Lemma VII. Let $f_{x}(P), P \in$ arc $P_{j} P_{i+1}$, be defined as $f_{x}(X(y), y)$ when the latter exists. $f_{x}(P), P \epsilon \operatorname{arc} P_{j} P_{j+1}$, is a measurable function of $s$ defined for almost every s corresponding to a point of $\operatorname{arc} P_{j} P_{j+1}$, and there is a $K$ such that

$$
\int_{\operatorname{aro} P_{j} P_{j+1}}\left|f_{x}(P)\right|^{2} d s \leqq K\|f\|^{2} .
$$

It is readily seen that $y(s)$ is an absolutely continuous function of $s$ with bounded Dini derivatives and that, conversely, $s(y)$ is such a function of $y$. Then in view of Lemma VII, (S) Lemma 6.4 (1) will imply by a well known result of the theory of changing the variable in a definite integral*

$$
\int_{\operatorname{aro} P_{j} P_{j+1}}\left|f_{x}(P)\right|^{2} d s=\int_{\operatorname{arc}_{j} P_{j+1}} D_{y} s\left|f_{x}(P)\right|^{2} d y \leqq K\|f\|^{2} .
$$

We have shown that for certain possible situations for cases (1) and (2) of the arcs of $B$, we may define $f_{x}(P), P \epsilon B$, for almost every value of $s$ in the appropriate interval, that $f_{x}(P)$ is measurable with respect to $s$ and $\left|f_{x}(P)\right|^{2}$ is summable and that its integral along a piece of the arc is less than a constant times $\|f\|^{2}$. Similar considerations hold for $f_{y}$ and $f$ and it is quite obvious that the other possibilities which may arise under cases (1) and (2) can be treated in a similar way, and since $B$ is made up of a finite number of such arcs which satisfy (1) or (2) we may conclude

Lemma VIII. When $f_{x}(P), f_{y}(P)$ and $f(P)$ are defined as the limits of $f_{x}$, $f_{y}$ and $f$ respectively along a line parallel to an axis in the manner described above, then $f_{x}(P), f_{y}(P), f(P)$ are measurable functions of $s$ defined for almost every $s$ corresponding to a point of $B$, with a summable square, and there exist constants $K_{x}, K_{y}$ and $K_{0}$ (independent of $f$ ) such that

$$
\begin{gathered}
\int_{B}\left|f_{x}(P)\right|^{2} d s \leqq K_{x}\|f\|^{2}, \int_{B}\left|f_{y}(P)\right|^{2} d s \leqq K_{y}\|f\|^{2}, \\
\int_{B}|f(P)|^{2} d s \leqq K_{0}\|f\|^{2} .
\end{gathered}
$$

* (S) I.emma 6.3. 


\section{Boundary VAlUE PROBLEM}

DeFinition V. Let the space \&5 be defined as the space of classes of functions defined on the boundary and having a summable square with respect to $s$, two functions $f^{\prime}$ and $g^{\prime}$ belonging to the same class if and only if

$$
\int_{B}\left|f^{\prime}-g^{\prime}\right|^{2} d s=0 .
$$

Addition and multiplication by a constant are to have their usual significance, and for $f^{\prime} \in f$ and $g^{\prime} \epsilon g,(f, g)=\int_{B} f^{\prime} g^{-1} d s$.

That (B) is a Hilbert space is a special case of (S) Theorem 1.24, when we take for $E$ the set, on the $s$ axis, which corresponds to points of $B$.

THEOREM IX. Let

$$
Q^{\prime} f^{\prime}=\beta_{1} f_{x}^{\prime}(P)+\beta_{2} f_{y}^{\prime}(P)+\beta_{8} f^{\prime}(P),
$$

where the $\beta$ 's are essentially bounded measurable functions of $s$ defined along $B$. Let $Q$ be the transformation from $\mathfrak{B}$ to $\Theta$, which is such that $\{f, g\} \in Q$, if and only if for all functions $f^{\prime} \epsilon H_{s}$ and $f^{\prime} \epsilon f, Q^{\prime} f^{\prime} \epsilon g$. Then $Q$ is a limited transformation with domain $\mathfrak{B}$.

Since the $\beta$ 's are essentially bounded, measurable functions if $f \in \mathfrak{B}$ is given, it follows from Lemma VII that for every $f^{\prime} \epsilon f, Q^{\prime} f^{\prime}$ exists and there is a $g \in\left(\mathbb{S}\right.$, such that $Q^{\prime} f^{\prime} \epsilon g$. Now if $f^{\prime \prime} \epsilon f$, then $f^{\prime}-f^{\prime \prime}={ }_{m+} 0$ and $f^{\prime}-f^{\prime \prime}$ is zero for almost every line parallel to an axis. Hence $Q^{\prime} f^{\prime}-Q^{\prime} f^{\prime \prime}={ }_{m} 0$ and $Q^{\prime} f^{\prime \prime} \epsilon g$. Thus the domain of $Q$ is $\mathfrak{B}$.

Now let $M$ be a measurable upper bound of the absolute values of $\beta_{i}$, $i=1,2,3$. Then

$$
\begin{aligned}
\|Q f\|^{2}= & \int_{B}\left|Q^{\prime} f^{\prime}\right|^{2} d s \leqq \int_{B}\left(\left|\beta_{1}\right|^{2}+\left|\beta_{2}\right|^{2}+\left|\beta_{3}\right|^{2}\right) \\
& \cdot\left(\left|f_{x}^{\prime}(P)\right|^{2}+\left|f_{y}^{\prime}(P)\right|^{2}+|f(P)|^{2}\right) d s \leqq 3 M^{2}\left(K_{x}+K_{y}+K_{0}\right)\|f\|^{2},
\end{aligned}
$$

and $Q$ is limited.

We now state our fundamental boundary value for which we can now give a method of solution.

Problem. Let $L(u)$ be as in the Introduction, $Q^{\prime}(u)$ as in the preceding Theorem, and let $\overline{\mathfrak{M}}\left(\Re_{0}\right)=\mathfrak{S}^{\prime}$. Let $v$ be a member of an element of $\Re, w$ a member of $\mathcal{B}^{\prime}$. Required to find all $u \in H_{s}$, such that

$$
L(u)={ }_{m} v, Q^{\prime}(u)=_{m} w .
$$

Let $E_{1}^{\prime}$ be the projection on the manifold of zeros of the transformation $T$ 
associated with $L$ and $E_{2}^{\prime}$ that on the manifold of zeros of $Q$. We can by applying Theorem II construct $I-E_{1}^{\prime}=E_{1}$ and $I-E_{2}^{\prime}=E_{2}$ by means of the method given in the note on construction, since $T$ and $Q$ are limited. On applying Theorem II again, we can state that either no $u$ satisfying (1) exists or $u$ belongs to an element of $\mathfrak{B}, f$, whose projections $E_{1} f$ and $E_{2} f$ we can calculate. Furthermore, we know that any $u$ which is in a class $f$ having the calculated projections satisfies (1).

Thus our problem becomes to find all elements $f$ whose projections on two manifolds $E_{1} f$ and $E_{2} f$ are given, when we know a complete orthonormal set in each manifold. Or given $E_{1}, E_{2}, f_{1}$ and $f_{2}$, find all $f$ such that

$$
\begin{aligned}
& E_{1} f=f_{1}, \\
& E_{2} f=f_{2} .
\end{aligned}
$$

From (a) we obtain

$$
f=f_{1}+\left(I-E_{1}\right) f
$$

Substituting in (b) yields

$$
E_{2}\left(I-E_{1}\right) f=f_{2}-E_{2} f_{1}
$$

Now consider the closed linear manifold $\mathfrak{M}$, determined by the range of $E_{2}\left(I-E_{1}\right)$. Since $E_{2}\left(I-E_{1}\right)$ is limited, we can, by using Theorem II, obtain an orthonormal set which determines $\mathfrak{M}$. Hence we can determine whether $f_{2}-E_{2} f_{1}$ is in $\mathfrak{M}$ or not. If $f_{2}-E_{2} f_{1}$ is not in $\mathfrak{M}$ then (2) has no solutions. If $f_{2}-E_{2} f_{1}$ is in $\mathfrak{M}$, since $E_{2}\left(I-E_{1}\right)$ is limited, taking $\mathfrak{M}$ as our range space, we can apply Theorems 1.16 and II and obtain all $f$ which satisfy (2). Since the manifold of zeros of $E_{2}\left(I-E_{1}\right)$ includes the range of $E_{1}$, we can find all $f$ which satisfy (a) and (2) or the equivalent set of equations (a) and (b).

If, in the above problem, $w$ is zero, we may shorten the above discussion by taking as our domain space for $T$ the closed linear manifold of all $f$ such that $Q f=0$. A similar remark applies, when $v$ is zero.

COLUmbia UnIVERSITY, NEW YORK, N.Y. 